\title{
Formfactors of Relativistic Composite Particle Interactions in Unified Nonlinear Spinorfield Models
}

\author{
H. Stumpf \\ Institut für Theoretische Physik, Universität Tübingen \\ Z. Naturforsch. 40 a, 752-774 (1985); received April 20, 1985
}

\begin{abstract}
Unified nonlinear spinorfield models are self-regularizing quantum field theories in which all observable (elementary and non-elementary) particles are assumed to be bound states of fermionic preon fields. Due to their large masses the preons themselves are confined and below the threshold of preon production the effective dynamics of the model is only concerned with bound state reactions. In preceding papers a functional energy representation, the statistical interpretation and the dynamical equations were derived and the effective dynamics for preonantipreon boson states and three preon-fermion states (with corresponding anti-fermions) was studied in the low energy limit. The transformation of the functional energy representation of the spinorfield into composite particle functional operators produced a hierarchy of effective interactions at the composite particle level, the leading terms of which are identical with the functional energy representation of a phenomenological boson-fermion coupling theory. In this paper these calculations are extended into the high energy range. This leads to formfactors for the composite particle interaction terms which are calculated in a rough approximation and which in principle are observable. In addition, the mathematical and physical interpretation of nonlocal quantum field theories and the meaning of the mapping procedure, its relativistic invariance etc. are discussed.
\end{abstract}

PACS 11.10 Field theory

PACS 12.10 Unified field theories and models

PACS 12.35 Composite models of particles

\section{Introduction}

Unified nonlinear spinorfield ( $\equiv$ NSF) models are quantum field theories in which all observable (elementary and non-elementary) particles are assumed to be bound states of elementary fermion fields. Accordingly, such models must be formulated by dynamical laws for self-coupled fermion fields only.

If self-coupled fermion fields are described by NSF equations with first order derivatives (三FDNSF) and local interactions the corresponding quantum field theories are non-renormalizable. To circumvent this non-renormalizability, higher order derivative nonlinear spinorfield ( equations can be used. These equations exhibit selfregularizing properties and are thus renormalizable or super-renormalizable. However, they lead to indefinite metric in the corresponding state spaces. A short survey of the literature concerning HDNSF equations can be found for instance in [1].

Using quantized HDNSF models, five different problems have to be treated in order to provide a

Reprint requests to Prof. Dr. H. Stumpf, Institut für Theoretische Physik der Universität Tübingen, Auf der Morgenstelle 14, D-7400 Tübingen. proper physical and mathematical basis for the application of such models:

i) the foundation of self-regularizing HDNSF equations themselves;

ii) the proof that in spite of the indefinite metric a meaningful physical and statistical interpretation of the theory is guaranteed;

iii) the derivation of the bound state spectrum;

vi) the proof that bound states representing "elementary particles" satisfy in certain approximations the corresponding gauge theories which govern the reactions of these particles if they are considered to be elementary and pointlike;

v) the derivation of corrections of conventional gauge theories which result from the non-elementarity of their "elementary" constituents.

Although numerous efforts were made in the past to treat NSF models, no satisfactory and systematic answers are available in the literature for the solution of these peculiar problems. Therefore, a research program was started by the author and collaborators in this field. The following results have been established so far:

Grosser and Lauxmann [2] proposed an NSF model with second order derivatives at the preon level. A decomposition theorem of HDNSF equa- 
tions into a set of FDNSF equations which allows the canonical quantization of HDNSF equations was proved by the author [3] and Grosser [4]. By application of this theorem a self-regularizing second order derivative equation was derived from an FDNSF equation through a symmetry breaking quantization by the author [5]. The same theorem was used for the derivation of a functional energy eigenvalue equation for the calculation of relativistic bound states etc. by Grosser, Hailer, Hornung, Lauxmann, and Stumpf [6]. For this energy representation the author [7] discussed the properties of solutions in the two-fermion sector and confirmed the possibility of giving a correct statistical interpretation of the theory in the case of very high preon masses. This method can easily be extended to higher fermion sectors. Thus, as far as the problems i), ii) and iii) are concerned, considerable progress has been made and further papers about these topics are in preparation. Therefore, at the present stage of the theory the problems iv) and v) need to be investigated.

The treatment of both these problems is very complicated. A first step in this direction was made in three preceding papers [8] which we denote by I, II, III in the following. In these papers composite particles were defined to be eigensolutions of the diagonal part of the functional energy representation of a second order derivative NSF model, and a suitable composite particle spectrum was assumed. It consists of preon-antipreon boson states and three-preon fermion states with corresponding antifermions and contains bound states as well as preon scattering states. The NSF energy functional states were expanded in terms of these composite particle states with inclusion of preon scattering states. Then the transformation of the functional NSF energy representation into composite particle functional operators produced a hierarchy of effective interactions at the composite particle level, the leading terms of which are identical with the functional energy representation of a phenomenological bosonfermion coupling theory. This representation is valid as long as the processes are assumed to be far below the energetic threshold for preon production or preon break-up reactions, respectively. In this paper we will complete this investigation and we will evaluate the formalism in particular with respect to problem v), i.e. we will investigate the high energy behavior of this map of a unified NSF- model on a boson-fermion coupling theory even at energies above the energetic threshold of preon break-up reactions.

While on the "low" energy level this map produced a functional energy representation of a boson-fermion coupling theory with local coupling, we will show in the following that for sufficiently high energies the boson-fermion coupling becomes nonlocal, i.e. formfactors will appear. The loss of locality and the appearance of formfactors in the boson-fermion coupling terms in the high-energy region need an explanation what kind of phenomenological theory we have really obtained by performing such a map. Before going into details we discuss this basic problem.

Owing to the above mentioned nonlocality we first raise the question whether a phenomenological nonlocal boson-fermion coupling theory can be considered as the result of such a map. A formfactor model for electrodynamics was first proposed by Wataghin [9]. Later on further such models were proposed by Bopp [10], McManus [11], Feynman [12] and de Broglie [13], while Yukawa [14] introduced bilocal fields. The quantization of these models offered great difficulties. Since the postulated Poincaré invariance enforces nonlocal couplings or bilocal fields to be spread out in space-time regions, no conventional Hamiltonian formalism exists which is needed for canonical quantization. To circumvent these difficulties Rayski [15] applied the Yang-Feldman-Källen quantization procedure to the McManus model. With the same method Kristensen and Möller [16] treated a nonlocalized Yukawa coupling. However, further difficulties were discovered concerning the existence of the $S$-matrix and the fulfillment of macrocausality conditions. Hayashi [17] found a violation of asymptotic completeness in the Kristensen-Möller model and Bloch [18], Chretien and Peierls [19] and Ebel [20] discussed restrictions due to macrocausality. The aim of all these theories was to get convergent perturbation expansions. However, this was not easily established in a satisfactory way, cf. Chretien and Peierls [21] and Möller [22]. In the further development of this approach it was tried to remove these difficulties. For brevity we can only cite the authors of the most important papers, namely Bloch [23], Fierz [24], Yennie [25], Rayski [26], Ascoli [27], Jordan, H. L. [28], Gulmanelly [29], Hara and Shimazu [30], Katayama [31], Pauli [32], Ono [33], 
Rzewuski [34], Wataghin [35], Budini and Vili [36], Kamefuchi [37], Gaus [38], Stueckelberg and Wanders [39], Taniuty [40], Sen [41], Budini [42], Minardi [43], Barashenkov [44], Medvedev [45], Weidlich [46], Nagy and Rzewuski [47], Arnous and Heitler [48], Heber [49], O’Raifeartaigh [50], Arnous, Heitler and Takahashi [51], Arnous, Heitler, and O'Raifeartaigh [52], Tani [53], Shcherbina [54], Sutula [55], O'Raifeartaigh and Takahashi [56], Kirzhnits [57], Pham Xuan Yem [58], Levy [59], Shimazu and Yokoyama [60], Leznov and Kirzhnits [61], Guillot, Jaus, and O'Raifeartaigh [62], Leznov [63], Priou [64], Efimov [65], Fraas [66], Yamamoto [67], Iofa and Fainberg [68], Prokhorov [69], Gallaher [70], La Camera and Wataghin [71], Nilsson [72], Efimov and Seltzer [73], Tran huu Phat [74], Alebastrov, Efimov and Seltzer [75], Alebastrov and Efimov [76], and Rivers [77].

Although interesting results were achieved within these three decades of research it was not possible to clarify all problems of nonlocal field theory. This is the conclusion which follows from the above mentioned papers but which cannot be discussed here in detail; cf. for instance two (incomplete) review articles [57] and [62]. The most successful approach was performed in [75] and [76] where a finite, unitary, causal and gauge invariant $S$-matrix with respect to a nonlocal electromagnetic and weak interaction was derived. However, these authors arrived at their results at the expense of taking for granted the expression for the $S$-matrix in the interaction representation of a canonical local quantum field theory. But it is just this assumption which needs a critical reexamination. This was demonstrated by Marnelius [78]. Within the framework of the Yang-Feldman-Källen quantization procedure Marnelius showed by a thorough investigation of the Kristensen-Möller model that no meaningful $S$-matrix can be defined at all and that this deficiency cannot be corrected either by restriction of the formfactor functions of by adding correction terms to the perturbation expansions. The relation of this result to the most successful papers [57], [75], [76] has not been clarified so far, but since that time no basic paper about nonlocal quantum field theory appeared.

An analysis of the Marnelius paper reveals that one can escape his conclusions only at the prize of sacrificing Poincaré invariance (and causality) by restricting the formfactor to spacelike hyperplanes (which was not the intention of this author). I.e. for such models one has either the $S$-matrix but no Poincaré invariance, or Poincaré invariance but no $S$-matrix which is likewise disastrous. Therefore a meaningful map should not lead to a nonlocal boson-fermion coupling theory, although formfactors appear in the interaction terms. The resolution of this difficulty follows from a consideration of the mapping itself.

In I it has been emphasized that in order to derive descriptions of composite particle dynamics and effective interactions all approaches can generally be grouped into two classes: the composite particle operator description and the composite particle state description. As this classification is basic for all further discussions about this topic it is useful to characterize these approaches more precisely. In analogy to the definitions of weak and strong operator convergence we introduce two definitions which are concerned with the map between field theories for "elementary" and composite particles.

Definition 1: A mapping between two field theories is called a strong mapping if it is defined by a homomorphism between the operator algebras of the two field theories and their corresponding dynamical equations.

Definition 2: A mapping between two field theories is called a weak mapping if it is defined by a projection between the state functionals of the two field theories and their corresponding dynamical equations.

From these definitions it is obvious that, applied to a given field theory, only strong mappings yield again field theories, while weak mappings need not necessarily lead to field theories. So far, strong mappings have been used in relativistic quantum field theory (including nonrelativistic models) while weak mappings (in Hilbert spaces) have mainly been used in nuclear physics and other branches of many body physics. With respect to strong mappings in I a short survey of the literature was given and we need not repeat it here. We only complete the list of authors wich have critisized these procedures, namely Broido [79], Lurie [80], La Camera and Wataghin [71], Kerler [81] (in agreement with Symanzik) Rajaraman [82], Eguchi [83], Tamvakis and Guralnik [84], Banks and Zaks [85], Chiang, 
Chiu, Sudarshan and Tata [86], Nakanishi [87], Ellwanger [88]. Their conclusions are: A strong mapping has not really been proved so far, leads to contradictions, or cannot exist at all. The most transparent paper is that of Rajaraman [82]. Apart from bosonization techniques which are of no interest here, he demonstrated the impossibility of strong mappings by the evaluation of path integrals.

But even if one succeeded in performing a strong mapping one would arrive at the next trap: The composite particle theories which are assumed to be the result of strong mappings are bound to be strictly local theories, otherwise they would not exist, as was discussed above. I.e. there would be no possibility to detect composite particle effects on the phenomenological level. It should be noted that this criticism does not deny the possibility of the construction of composite field operators themselves and their use for scattering calculations, operator expansions etc. What is merely rejected is their use in connection with strong mappings.

To circumvent these difficulties with strong mapping, in I the approach to weak mapping in relativistic quantum field theories was initiated which is a non-trivial functional analogon to the weak mapping methods elaborated in nuclear physics among others by Wildermuth [89], Schmid [90], Kramer [91], and in nuclear quark physics by Faessler [92], Müther [93], Bräuer [94], etc. By this method functional equations for composite particle dynamics and effective interactions can be derived which coincide only for "low" energies with the functional state equations of the corresponding phenomenological coupling theories where the composite particles are assumed to be.pointlike, while they differ from those equations in "high" energy regions. For such functional state equations no corresponding relativistic operator quantum field theories exist which are valid in all energy ranges and satisfy all axioms which are usually required for these theories. Rather such theories are only simulated by the functional state equations in certain energy ranges. At the level of composite particles no operators, no commutation relations, no microcausality, no locality and even no explicit Poincaré invariance are required. All these properties are only required for the fundamental field and are satisfied by it. In this way the weak mapping avoids all the difficulties which are connected with strong mapping, and in addition it avoids the difficulties which are connect- ed with local and nonlocal operator quantum field coupling theories, for instance infinities etc., as were already discussed. That means that from the point of view of weak mapping procedures the known relativistic quantum field coupling theories for pointlike (but truly composite) particles are only extrapolations. In the case of local couplings one has to pay the prize for this extrapolation by the infinite renormalization constants, in the case of nonlocal couplings by the nonexistence of the $S$-matrix.

The application of the weak mapping procedure thus implies that really only one correct quantum field theory exists, namely that of the fundamental field to which all problems of invariance, microcausality etc. must be traced back. However, this procedure implies too, that on the composite particle level all physical information must be obtainable from the functional state equations alone, as on this level the existence of quantum field coupling theories is considered only as an extrapolation which cannot be used by the "true" theory. The possibility of establishing a complete quantum theory only by means of functional states and corresponding functional state equations was confirmed by the author in the development of the functional quantum theory [95]. Once the functional equations are given one can forget about their origin (which commonly stems from an operator field theory) and can find all physically relevant answers only from these equations. In our case such equations arise by weak mappings which is, however, without relevance for the application of the formalism of functional quantum theory. This formalism can also be used without the background of an operator field theory.

Summarizing the properties of weak mappings we may say that weak mappings allow to recognize the phenomenological particle dynamics of composite particles whose reactions are governed by a fundamental field theory, but that at the same time the drawbacks of strong mappings themselves as well as those of the phenomenological coupling theories are avoided. On the other hand, with the non-existence of a field operator algebra at the phenomenological (composite) particle level which satisfies the common requirements of local relativistic quantum field theories, some problems like unitary etc. are more difficult to handle. So sometimes it may be of advantage to substitute an auxiliary field operator algebra which does not 
fulfill all requirements of a local relastivistic quantum field theory, but which imitates the results of functional quantum theory in a limited energy range etc. This is then a problem of the detailed evaluation and interpretation of functional quantum theory and does not touch the basic approach itself.

In the following we treat the quantization problem, the high energy behavior and the problem of relativistic invariance with respect to the weak mapping of a unified nonlinear spinorfield model which was defined in I and other related papers. For brevity in this introduction we forgo a detailed description of the content of the following sections and restrict ourselves to the discussion of the basic interpretation problems of such mappings which was given above.

\section{Coupling Theory Energy Representation}

For comparison with the results of a weak mapping of a nonlinear spinorfield on to a bosonfermion coupling theory we derived in I the functional energy representation of a corresponding phenomenological coupling theory. In order to get a first and foremost information about this map we did not take special care of the conditions which need to be imposed upon this transition to the energy representation. In this paper we will remove this deficiency at least partially.

As was shown by Maison and Stumpf [96] for the case of the anharmonic oscillator a necessary and sufficient condition for performing the limit to equal times in the energy representation is the continuity of the coefficient functions of the state functional in all time variables. Time ordered coefficients do not have this property. Hence the time ordered state functionals must be transformed in such a way that the discontinuous parts are separated from the continuous parts in their coefficient functions. In the case of the anharmonic oscillator this can be achieved by a simple normal ordering. In the case of a quantum field theory the normal ordering must be generalized to include the propagators of all bound states which occur in the theory, as any bound state propagator gives rise to such time variable discontinuities.

For brevity we assume that the phenomenological fermion-boson coupling theory does not possess any bound state. Then we can perform a simple normal ordering in analogy to the anharmonic oscillator. Having done this it is obvious how to proceed in more complicated cases, i.e. if bound states are admitted to occur. As will be seen in the following the transition to normal transforms removes those terms in the functional equations which stem from the field quantization, i.e. from the field commutation relations. The field quantization is then indirectly contained in the propagators. This was already indicated in III.

According to I the phenomenological field equations are given by

$$
\begin{aligned}
& \left(i \gamma^{\mu} \partial_{\mu}-m\right)_{\alpha \beta} \chi_{\beta}(x)=i g \Gamma_{\alpha \beta} \chi_{\beta}(x) \vartheta(x), \\
& \left(\partial^{\mu} \partial_{\mu}-\mu^{2}\right) \vartheta(x)=-i g \bar{\chi}_{\alpha}(x) \Gamma_{\alpha \beta} \chi_{\beta}(x)
\end{aligned}
$$

with $\%(x)$ the phenomenological fermion field and $z(x)$ the phenomenological boson field.

The quantum version of this theory is defined by state functionals $\mathfrak{I}[\eta, \bar{\eta}, J, a]\rangle$ in which $\eta_{x}(x)$, $\bar{\eta}_{x}(x)$ and $J(x)$ are sources of the $\chi, \bar{\chi}, \vartheta$ fields, respectively, and $|a\rangle$ denotes the quantum state of the system. The definition of state functionals for this model and the derivation of their corresponding functional equations are given for instance by Lurie [80]. These equations read

$$
\begin{aligned}
& \left(i \gamma^{\mu} \partial_{\mu}-m\right)_{\alpha \beta} \frac{\delta}{\delta \eta_{\beta}(x)}|\mathfrak{I}\rangle \\
& \quad=i \bar{\eta}_{x}(x)|\mathfrak{I}\rangle+g \Gamma_{\alpha \beta} \frac{\delta}{\delta \eta_{\beta}(x)} \frac{\delta}{\delta J(x)}|\mathfrak{I}\rangle
\end{aligned}
$$

and

$$
\begin{aligned}
& \left(\partial^{\mu} \partial_{\mu}-\mu^{2}\right) \frac{\delta}{\delta J(x)}|\mathfrak{T}\rangle \\
& \quad=-i J(x)|\mathfrak{T}\rangle-g \frac{\delta}{\delta \bar{\eta}_{x}(x)} \Gamma_{\alpha \beta} \frac{\delta}{\delta \eta_{\beta}(x)}|\mathfrak{T}\rangle
\end{aligned}
$$

if for brevity we omit the equation with respect to $\bar{\eta}(x)$ here and in the following.

The first terms on the right-hand side of (1.2) and (1.3) are the quantization terms which will be removed by normal-ordering. The corresponding transformation is defined by

$$
\mathfrak{I}\rangle=Z_{0}[\eta, \bar{\eta}, J]|\mathfrak{\mho}\rangle
$$

with

$$
\begin{aligned}
Z_{0}[\eta, \bar{\eta}, J] & :=\exp \left[-\int \eta_{x}(x) S_{\alpha \beta}^{\mathrm{F}}\left(x-x^{\prime}\right) \eta_{\beta}\left(x^{\prime}\right) \mathrm{d}^{4} x \mathrm{~d}^{4} x^{\prime}\right. \\
& \left.-\frac{1}{2} \int J(x) \Delta^{\mathrm{F}}\left(x-x^{\prime}\right) J\left(x^{\prime}\right) \mathrm{d}^{4} x \mathrm{~d}^{4} x^{\prime}\right],
\end{aligned}
$$


where $S^{\mathrm{F}}$ and $\Delta^{\mathrm{F}}$ are the causal Greenfunctions of the free fermion and boson equations of (1.1) respectively. Substitution of (1.4) into (1.2) and (1.3) yields

$$
\begin{aligned}
& \left(i \gamma^{\mu} \partial_{\mu}-m\right)_{\alpha \beta} \frac{\delta}{\delta \eta_{\beta}(x)}|\mathfrak{F}\rangle \\
& =g\left[-\int \Delta^{\mathrm{F}}\left(x-x^{\prime}\right) J\left(x^{\prime}\right) \mathrm{d}^{4} x^{\prime}+\frac{\delta}{\delta J(x)}\right] \Gamma_{\alpha \beta}\left[-\int \bar{\eta}_{x}\left(x^{\prime}\right) S_{\alpha \beta}^{\mathrm{F}}\left(x^{\prime}-x\right) \mathrm{d}^{4} x^{\prime}+\frac{\delta}{\delta \eta_{\beta}(x)}\right]|\mathfrak{F}\rangle, \\
& \left(\partial^{\mu} \partial_{\mu}-\mu^{2}\right) \frac{\delta}{\delta J(x)}|\mathfrak{F}\rangle \\
& \left.=-g\left[-\int S_{\alpha \beta}^{\mathrm{F}}\left(x-x^{\prime}\right) \eta_{\beta}\left(x^{\prime}\right) \mathrm{d}^{4} x^{\prime}+\frac{\delta}{\delta \bar{\eta}_{\alpha}(x)}\right] \Gamma_{\alpha \beta}\left[-\int \bar{\eta}_{x}\left(x^{\prime}\right) S_{\alpha \beta}^{\mathrm{F}}\left(x^{\prime}-x\right) \mathrm{d}^{4} x^{\prime}+\frac{\delta}{\delta \eta_{\beta}(x)}\right] \mathfrak{F}\right\rangle .
\end{aligned}
$$

We now proceed analogously to I. We introduce the conjugate momentum $\pi$ of the $\vartheta$-field and its corresponding source operator $K(x)$. Then we go over to the functional energy representation. After having done this the energy functional equation only depends on a single time parameter $t$. Without loss of generality we can assume this parameter to be zero. Performing then a Fourier-Transformation of the resulting functional equation and applying the transformation

$$
\tilde{K}(\boldsymbol{p})=2^{-1 / 2} \omega(\boldsymbol{p})^{-1 / 2}\left[b_{-}(\boldsymbol{p})-b_{+}(-\boldsymbol{p})\right], \quad \tilde{J}(\boldsymbol{p})=2^{-1 / 2} \omega(\boldsymbol{p})^{1 / 2}\left[b_{-}(\boldsymbol{p})+b_{+}(\boldsymbol{p})\right],
$$

we eventually obtain the equation

$$
\begin{aligned}
& E|\mathfrak{F}\rangle=\int \eta_{\alpha}(\boldsymbol{q}) h_{\alpha \beta}(\boldsymbol{q}) \frac{\delta}{\delta \eta_{\beta}(\boldsymbol{q})} \mathrm{d} q|\mathfrak{F}\rangle+\int \bar{\eta}_{\alpha}(\boldsymbol{q}) \bar{h}_{\alpha \beta}(\boldsymbol{q}) \frac{\delta}{\delta \bar{\eta}_{\beta}(\boldsymbol{q})} \mathrm{d} q|\mathfrak{F}\rangle \\
& +\int \omega(\boldsymbol{q})\left[b_{-}(\boldsymbol{q}) \frac{\delta}{\delta b_{-}(\boldsymbol{q})}-b_{+}(\boldsymbol{q}) \frac{\delta}{\delta b_{+}(\boldsymbol{q})}\right] \mathrm{d} q|\mathfrak{F}\rangle+g \int 2^{-1 / 2} \omega(\boldsymbol{k})^{-1 / 2}\left[\frac{\delta}{\delta b_{-}(\boldsymbol{k})}+\frac{\delta}{\delta b_{+}(\boldsymbol{k})}\right] \\
& {\left[\eta_{\alpha}(\boldsymbol{q}) \hat{\Gamma}_{\alpha \beta} \frac{\delta}{\delta \eta_{\beta}\left(\boldsymbol{q}^{\prime}\right)}-\bar{\eta}_{\beta}(\boldsymbol{q}) \hat{\hat{\Gamma}}_{\alpha \beta} \frac{\delta}{\delta \bar{\eta}_{\alpha}\left(\boldsymbol{q}^{\prime}\right)}\right] \delta\left(\boldsymbol{k}+\boldsymbol{q}^{\prime}-\boldsymbol{q}\right) \mathrm{d} k \mathrm{~d} q \mathrm{~d} q^{\prime}|\mathfrak{F}\rangle} \\
& -g \int 2^{-1 / 2} \omega(\boldsymbol{k})^{-1 / 2}\left[b_{-}(\boldsymbol{k})-b_{+}(-\boldsymbol{k})\right] \frac{\delta}{\delta \bar{\eta}_{\alpha}(\boldsymbol{q})} \Gamma_{\alpha \beta} \frac{\delta}{\delta \eta_{\beta}\left(\boldsymbol{q}^{\prime}\right)} \delta\left(\boldsymbol{k}-\boldsymbol{q}-\boldsymbol{q}^{\prime}\right) \mathrm{d} k \mathrm{~d} q \mathrm{~d} q^{\prime}|\mathfrak{F}\rangle \\
& +g \int 2^{-1 / 2} \omega(\boldsymbol{k})^{1 / 2} \Delta(\boldsymbol{k})\left[b_{-}(\boldsymbol{k})+b_{+}(-\boldsymbol{k})\right]\left[\eta_{\alpha}(\boldsymbol{q}) \hat{\Gamma}_{\alpha \beta} \frac{\delta}{\delta \eta_{\beta}\left(\boldsymbol{q}^{\prime}\right)}+\bar{\eta}_{\beta}(\boldsymbol{q}) \hat{\hat{\Gamma}}_{\alpha \beta} \frac{\delta}{\delta \bar{\eta}_{\beta}\left(\boldsymbol{q}^{\prime}\right)}\right] \\
& \cdot \delta\left(\boldsymbol{k}+\boldsymbol{q}-\boldsymbol{q}^{\prime}\right) \mathrm{d} k \mathrm{~d} q \mathrm{~d} q^{\prime}|\mathfrak{F}\rangle-g \int 2^{-1 / 2} \omega(\boldsymbol{k})^{-1 / 2}\left[\frac{\delta}{\delta b_{-}(\boldsymbol{k})}+\frac{\delta}{\delta b_{+}(-\boldsymbol{k})}\right] \\
& \cdot\left[\eta_{x}(\boldsymbol{q}) \hat{\Gamma}_{\alpha \beta} S_{\beta \varkappa}^{\mathrm{T}}\left(-\boldsymbol{q}^{\prime}\right) \bar{\eta}_{\varkappa}\left(\boldsymbol{q}^{\prime}\right)-\bar{\eta}_{\beta}(\boldsymbol{q}) S_{\varkappa \alpha}^{\mathrm{T}}\left(\boldsymbol{q}^{\prime}\right) \hat{\hat{\Gamma}}_{\alpha \beta} \eta_{\varkappa}\left(\boldsymbol{q}^{\prime}\right)\right] \delta\left(\boldsymbol{k}-\boldsymbol{q}-\boldsymbol{q}^{\prime}\right) \mathrm{d} k \mathrm{~d} q \mathrm{~d} q^{\prime}|\mathfrak{F}\rangle \\
& +g \int 2^{-1 / 2} \omega(\boldsymbol{k})^{1 / 2}\left[b_{-}(\boldsymbol{k})+b_{+}(-\boldsymbol{k})\right] \Delta(\boldsymbol{k})\left[\eta_{x}(\boldsymbol{q}) \hat{\Gamma}_{\alpha \beta} S_{\beta \varkappa}^{\mathrm{T}}\left(-\boldsymbol{q}^{\prime}\right) \bar{\eta}_{x}\left(\boldsymbol{q}^{\prime}\right)-\bar{\eta}_{\beta}(\boldsymbol{q}) S_{x \alpha}^{\mathrm{T}}\left(\boldsymbol{q}^{\prime}\right) \hat{\hat{\Gamma}}_{\alpha \beta} \eta_{*}\left(\boldsymbol{q}^{\prime}\right)\right] \\
& \cdot \delta\left(\boldsymbol{k}+\boldsymbol{q}+\boldsymbol{q}^{\prime}\right) \mathrm{d} k \mathrm{~d} q \mathrm{~d} q^{\prime}|\mathfrak{F}\rangle+g \int 2^{-1 / 2} \omega(\boldsymbol{k})^{-1 / 2}\left[b_{-}(\boldsymbol{k})-b_{+}(-\boldsymbol{k})\right] \\
& \cdot\left[\eta_{\varkappa}(\boldsymbol{q}) S_{\varkappa \alpha}^{\mathrm{T}}(\boldsymbol{q}) \Gamma_{\alpha \beta} \frac{\delta}{\delta \eta_{\beta}\left(\boldsymbol{q}^{\prime}\right)}+\frac{\delta}{\delta \bar{\eta}_{\chi}\left(\boldsymbol{q}^{\prime}\right)} \Gamma_{\alpha \beta} S_{\beta \varkappa}^{\mathrm{T}}(-\boldsymbol{q}) \bar{\eta}_{\varkappa}(\boldsymbol{q})\right] \delta\left(\boldsymbol{k}+\boldsymbol{q}+\boldsymbol{q}^{\prime}\right) \mathrm{d} k \mathrm{~d} q \mathrm{~d} q^{\prime}|\mathfrak{F}\rangle \\
& -g \int 2^{-1 / 2} \omega(\boldsymbol{k})^{-1 / 2}\left[b_{-}(\boldsymbol{k})-b_{+}(-\boldsymbol{k})\right] \eta_{\varkappa}(\boldsymbol{q}) S_{\varkappa \alpha}^{\mathrm{T}}(\boldsymbol{q}) \Gamma_{\alpha \beta} S_{\beta \lambda}^{\mathrm{T}}\left(-\boldsymbol{q}^{\prime}\right) \bar{\eta}_{\lambda}\left(\boldsymbol{q}^{\prime}\right) \delta\left(\boldsymbol{k}+\boldsymbol{q}+\boldsymbol{q}^{\prime}\right) \mathrm{d} k \mathrm{~d} q \mathrm{~d} q^{\prime}|\mathfrak{F}\rangle
\end{aligned}
$$

with $\mathrm{d} q \equiv \mathrm{d}^{3} q$ etc., $\hat{\Gamma}=\gamma^{0} \Gamma, \hat{\hat{\Gamma}}=\Gamma \gamma^{0}$ and $h_{\alpha \beta}(\boldsymbol{q})$ the Dirac energy operator. The propagators $\Delta(\boldsymbol{q})$ and $S(\boldsymbol{q})$ appearing in (1.9) are the Fourier transforms of the equal time limes procedure with respect to $\Delta^{\mathrm{F}}$ and $S^{\mathrm{F}}$. These functions are given by

$$
\Delta^{\mathrm{F}}(x)=\Theta\left(x_{0}\right) i \Delta^{(+)}(x)-\Theta\left(-x_{0}\right) i \Delta^{(-)}(x), \quad S^{\mathrm{F}}(x)=-\Theta\left(x_{0}\right) i S^{(+)}(x)+\Theta\left(-x_{0}\right) i S^{(-)}(x) .
$$

The transition to equal times is symmetric in the boson time coordinates but antisymmetric in the fermion time coordinates, cf. Maison and Stumpf [96]. 
Hence we obtain with

$$
\begin{aligned}
& \operatorname{Lim}_{\mathrm{B}}:=\lim _{x_{0} \rightarrow+0}+\lim _{x_{0} \rightarrow-0}, \\
& \operatorname{Lim}_{\mathrm{F}}:=\lim _{x_{0} \rightarrow+0}-\lim _{x_{0} \rightarrow-0}
\end{aligned}
$$

the relations

$$
\begin{aligned}
& \operatorname{Lim}_{\mathrm{B}} \Delta^{\mathrm{F}}(x)=2 i \Delta^{(+)}(x), \\
& \operatorname{Lim}_{\mathrm{F}} S^{\mathrm{F}}(x)=-i S(x),
\end{aligned}
$$

where $\Delta^{(+)}(x)$ is the boson field commutator while $S(x)$ is the fermion free field anticommutator. Then $\Delta(\boldsymbol{k}):=\mathscr{F} 2 i \Delta^{(+)}(\boldsymbol{x})$ and $S(\boldsymbol{k}):=\mathscr{F}(-i) S(\boldsymbol{x})$ are the Fouriertransforms of these functions.

\section{High Momentum Boson Wave Functions}

The transition from a low energy range into a high energy range in the weak mapping of spinorfields on to coupling theories implies that at least some of the composite particles must be assumed to have high kinetic energies, i.e. large momenta. For the theoretical description we thus need the corresponding high momentum wave functions. Postponing the problem of the relativistic transformation properties of such states to Sect.5, we simply calculate the high momentum wave functions by means of the eigenvalue equation for composite particles of the spinorfield.

According to I the eigenvalue equation for composite particles of the spinorfield is given by definition by the equation

$$
\begin{aligned}
E \tilde{\mho}\rangle & =\sum_{r=1}^{2} \int j_{\varepsilon}^{r}(\boldsymbol{r}) G_{\varepsilon \chi}^{0}\left(i \boldsymbol{G}_{\alpha \beta} \cdot \nabla-m_{r} \delta_{\alpha \beta}\right) \partial_{\beta}^{r}(\boldsymbol{r}) \mathrm{d} r|\mathfrak{\mho}\rangle \\
& +g \int\left[j_{\varepsilon}^{1}(\boldsymbol{r})-j_{\varepsilon}^{2}(\boldsymbol{r})\right] G_{\varepsilon \chi}^{0} W_{\alpha \beta \gamma \delta} \\
\cdot & {\left[\sum_{r=1}^{2} F_{\beta \beta^{\prime}}^{r}\left(\boldsymbol{r}-\boldsymbol{r}^{\prime}\right) j_{\beta^{\prime}}^{r}\left(\boldsymbol{r}^{\prime}\right)\right]\left[\sum_{r^{\prime} r^{\prime \prime}=1}^{2} \partial_{\gamma}^{r^{\prime}}(\boldsymbol{r}) \partial_{\delta}^{r^{\prime \prime}}(\boldsymbol{r})\right] \mathrm{d} r \mathrm{~d} r^{\prime}|\mathfrak{\mho}\rangle }
\end{aligned}
$$

For the definition of the quantities occurring in (2.1) we refer to I. The boson states under consideration are assumed to be bound preon-antipreon states and their general state functional can be written

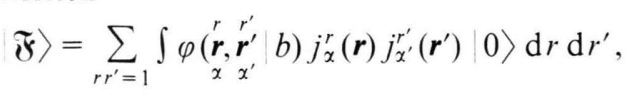

where $r$ and $r^{\prime}$ are the auxiliary field indices. For the further evaluation in [7] and II an orthogonal transformation of the sources with respect to the auxiliary fields was introduced. This transformation was explicitly given in [7] and II and induces in the space of boson wavefunctions (2.2) the transformation

$$
\left(\begin{array}{l}
\varphi^{11} \\
\varphi^{21} \\
\varphi^{12} \\
\varphi^{22}
\end{array}\right)=\frac{1}{2}\left(\begin{array}{rrrr}
1 & 1 & 1 & 1 \\
1 & -1 & 1 & -1 \\
1 & 1 & -1 & -1 \\
1 & -1 & -1 & 1
\end{array}\right)\left(\begin{array}{c}
\varphi^{\varrho \varrho} \\
\varphi^{\chi \varrho} \\
\varphi^{\varrho \chi} \\
\varphi^{\chi \chi}
\end{array}\right)
$$

where only the auxiliary field indices are used while all other indices etc. are omitted for brevity. The transformed wave functions themselves are not independent. Rather one has to employ the antisymmetric combination [7], II

$$
a=2^{-1 / 2}\left(\varphi^{\chi \varrho}+\varphi^{\varrho \chi}\right),
$$

whereas the corresponding symmetric combination has to be identical zero in order to get proper solutions of the eigenvalue equation (2.1). The remaining functions are

$$
\varphi_{1}:=\varphi^{\varrho} ; \quad \varphi_{2}:=a ; \quad \varphi_{3}:=\varphi^{\chi \chi} .
$$

For these functions in [7] from (2.1) a set of three equations was derived which can be written in the symbolical form

$$
\left(\begin{array}{crr}
3_{2} & -\mathfrak{M}_{2} & 0 \\
-\mathfrak{M}_{2}+\mathfrak{B}_{2} & 2 \mathfrak{Z}_{2} & -\mathfrak{M}_{2} \\
\mathfrak{B}_{2} & -\mathfrak{M}_{2} & 3_{2}
\end{array}\right)\left(\begin{array}{l}
\varphi_{1} \\
\varphi_{2} \\
\varphi_{3}
\end{array}\right)=0
$$

where the definitions of the symbols $3_{2}, \mathfrak{M}_{2}, \mathfrak{B}_{2}, \mathfrak{M}_{2}$ are given in [7]. The set of equations (2.6) can be resolved with respect to $\varphi_{2}$ and $\varphi_{3}$ in terms of $\varphi_{1}$ by

$$
\varphi_{2}=\mathfrak{M}_{2}^{-1} 3_{2} \varphi_{1}
$$

and

$$
\varphi_{3}=\left(2 \mathfrak{M}_{2}^{-1} 3_{2} \mathfrak{M}_{2}^{-1} \mathfrak{3}_{2}-1+\mathfrak{M}_{2}^{-1} \mathfrak{B}_{2}\right) \varphi_{1}
$$

and leads to the equation

$$
\begin{aligned}
\left(-23_{2}+2\right. & 3_{2} \mathfrak{M}_{2}^{-1} 3_{2} \mathfrak{M}_{2}^{-1} \mathfrak{Z}_{2} \\
& \left.+3_{2} \mathfrak{M}_{2}^{-1} \mathfrak{B}_{2}+\mathfrak{B}_{2}\right) \varphi_{1}=0
\end{aligned}
$$

for $\varphi_{1}$ alone. In II a rough approximation for (2.7) and (2.8) was performed in the low energy range in order to simplify the subsequent calculations required for the weak mapping procedure. In this 
paper we will again perform a suitable approximation for (2.7) and (2.8) but now with respect to the high energy range, i.e. large center of mass momenta of the corresponding bosons.

For a more detailed discussion of (2.7), (2.8), and (2.9) it is necessary to expand $\varphi_{1}, \varphi_{2}, \varphi_{3}$ in terms of Dirac spinors. For the case of a preon-antipreon bound state such an expansion is given by

$$
\begin{aligned}
\varphi_{i}\left(\underset{\beta}{\boldsymbol{r}, \boldsymbol{r}^{\prime}} b\right)=\sum_{s s^{\prime}} \int \tilde{\varphi}_{i}\left(\boldsymbol{k} s, \boldsymbol{k}^{\prime} s^{\prime} b\right) u_{\beta}(\boldsymbol{k} s) \\
\cdot v_{\beta^{\prime}}^{+}\left(\boldsymbol{k}^{\prime} s^{\prime}\right) e^{i \boldsymbol{k} \boldsymbol{r}-i \boldsymbol{k}^{\prime} \boldsymbol{r}^{\prime}} \mathrm{d} k \mathrm{~d} k^{\prime},
\end{aligned}
$$

$i=1,2,3$. The evaluation of (2.6) or (2.7), (2.8), and (2.9) respectively in terms of Dirac spinors according to the expansion (2.10) was performed by Hailer [97] and Hornung [98]. Thereby (2.7), (2.8), and (2.9) go over into the set of equations

$\tilde{\varphi}_{2}=M_{2}^{-1} Z_{2} \tilde{\varphi}_{1}$,

$\tilde{\varphi}_{3}=\left(2 M_{2}^{-1} Z_{2} M_{2}^{-1} Z_{2}-1+M_{2}^{-1} V_{2}\right) \tilde{\varphi}_{1}$,

$\left(-2 Z_{2}+2 Z_{2} M_{2}^{-1} Z_{2} M_{2}^{-1} Z_{2}\right.$

$$
\left.+Z_{2} M_{2}^{-1} V_{2}+W_{2}\right) \tilde{\varphi}_{1}=0
$$

with

$Z_{2} \equiv Z\left(\boldsymbol{p}, \boldsymbol{p}^{\prime}\right):=\left[E-E(\boldsymbol{p})-E\left(\boldsymbol{p}^{\prime}\right)\right]$,

$M_{2} \equiv M\left(\boldsymbol{p}, \boldsymbol{p}^{\prime}\right):=\Delta m M\left[E(\boldsymbol{p})^{-1}+E\left(\boldsymbol{p}^{\prime}\right)^{-1}\right]$

and

$E(\boldsymbol{p}):=\left(M^{2}+\boldsymbol{p}^{2}\right)^{1 / 2} ; \quad E:=\left[m_{b}^{2}+\left(\boldsymbol{p}+\boldsymbol{p}^{\prime}\right)^{2}\right]^{1 / 2} ;$

$\Delta m:=\frac{1}{2}\left(m_{1}-m_{2}\right) ; \quad M:=\frac{1}{2}\left(m_{1}+m_{2}\right)$,

where $m_{1}$ and $m_{2}$ are the large masses of the auxiliary fields occurring in (2.1) and $m_{b}$ the boson mass. The quantities $V_{2}$ and $W_{2}$ are integral kernels which for brevity are not explicitly given here.

The states (2.2) are the right-hand solutions of (2.1). For the complete description of the boson states including normalization etc. we also need the left-hand solutions of (2.1). For the boson states under consideration these left-hand solutions have the general form

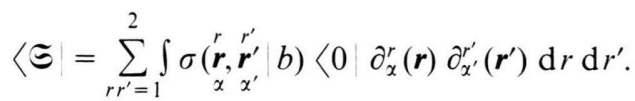

The treatment of (2.18) runs along the same lines as that of (2.2). In particular the set $\left\{\sigma^{i k}\right\}$ is subjected to the same transformation (2.3) as the set $\left\{\varphi^{i k}\right\}$, cf. II. Applying the symmetry combinations we then obtain three independent $\sigma$-functions

$$
\sigma_{1}:=\sigma^{\varrho \varrho} ; \quad \sigma_{2}:=\chi ; \quad \sigma_{3}:=\sigma^{\chi \chi}
$$

with $\chi:=(2)^{-1 / 2}\left(\sigma^{\chi \varrho}+\sigma^{\varrho \chi}\right)$. These functions are the left-hand solutions of (2.6). Resolution of these equations with respect to $\sigma_{1}$ and $\sigma_{2}$ in terms of $\sigma_{3}$ yields

$$
\sigma_{2}=\sigma_{3} \mathfrak{3}_{2} \mathfrak{M}_{2}^{-1}
$$

and

$$
\sigma_{1}=\sigma_{3}\left(2 \mathfrak{3}_{2} \mathfrak{M}_{2}^{-1} \mathfrak{3}_{2} \mathfrak{M}_{2}^{-1}-1\right),
$$

and leads to the equation

$$
\begin{aligned}
\sigma_{3}\left(-2 \mathfrak{Z}_{2}+\right. & 2 \mathfrak{3}_{2} \mathfrak{M}_{2}^{-1} \mathfrak{Z}_{2} \mathfrak{M}_{2}^{-1} \mathfrak{Z}_{2} \\
& \left.+\mathfrak{3}_{2} \mathfrak{M}_{2}^{-1} \mathfrak{B}_{2}+\mathfrak{W}_{2}\right)=0
\end{aligned}
$$

for $\sigma_{3}$ alone.

The expansion of the set $\left\{\sigma_{i}\right\}$ in terms of Dirac spinors is defined by

$$
\begin{aligned}
\underset{\beta}{\sigma_{i}}\left(\underset{\boldsymbol{r}}{\boldsymbol{r}^{\prime}} \boldsymbol{r}^{\prime} b\right)=\sum_{s s^{\prime}} \int \tilde{\sigma}_{i}\left(\boldsymbol{k} s, \boldsymbol{k}^{\prime} s^{\prime} \mid b\right) u_{\beta}^{+}(\boldsymbol{k} s) \\
\cdot v_{\beta^{\prime}}\left(\boldsymbol{k}^{\prime} s^{\prime}\right) e^{-\boldsymbol{k} \boldsymbol{r}+i \boldsymbol{k}^{\prime} \boldsymbol{r}^{\prime}} \mathrm{d} k \mathrm{~d} k^{\prime}
\end{aligned}
$$

and yields for (2.20), (2.21) and (2.22)

$$
\begin{aligned}
& \tilde{\sigma}_{2}=\tilde{\sigma}_{3} Z_{2} M_{2}^{-1}, \\
& \begin{aligned}
& \tilde{\sigma}_{1}=\tilde{\sigma}_{3}\left(2 Z_{2} M_{2}^{-1} Z_{2} M_{2}^{-1}-1\right), \\
& \tilde{\sigma}_{3}\left(-2 Z_{2}+2 Z_{2} M_{2}^{-1} Z_{2} M_{2}^{-1} Z_{2}\right. \\
& \\
&\left.\quad+Z_{2} M_{2}^{-1} V_{2}+W_{2}\right)=0 .
\end{aligned}
\end{aligned}
$$

These equations correspond to (2.11), (2.12) and (2.13) and will be used for appropriate approximations.

We now proceed to perform these approximations. We introduce center of mass momentum coordinates by

$$
\begin{array}{ll}
\boldsymbol{P}=\boldsymbol{p}+\boldsymbol{p}^{\prime} ; & \boldsymbol{p}=\frac{1}{2}(\boldsymbol{P}+\boldsymbol{u}) \\
\boldsymbol{u}=\boldsymbol{p}-\boldsymbol{p}^{\prime} ; & \boldsymbol{p}^{\prime}=\frac{1}{2}(\boldsymbol{P}-\boldsymbol{u})
\end{array}
$$

and assume that all bound state wavefunctions $\left\{\tilde{\varphi}_{i}\right\}$ or $\left\{\tilde{\sigma}_{i}\right\}$ are sufficiently concentrated about the origin of the relative momentum $\boldsymbol{u}$, i.e. can be considered as testfunctions with respect to $\boldsymbol{u}$, a fact that merely expresses their bound state properties. Then we consider the limit of very large center of mass momenta $\boldsymbol{P}$ with respect to (2.11). By applying the usual techniques of power series expansions etc. and observing the testfunction properties of $\left\{\tilde{\varphi}_{i}\right\}$ with 
respect to $\boldsymbol{u}$, we obtain for $(2.11)$

$$
\begin{aligned}
\lim _{P \rightarrow \infty} \tilde{\varphi}_{2} & =\lim _{P \rightarrow \infty} M_{2}^{-1} Z_{2} \tilde{\varphi}_{1} \\
& =\lim _{P \rightarrow \infty}\left(\frac{1}{4} \frac{P}{\Delta m M}\right)\left(-\frac{2 M^{2}}{P}\right) \tilde{\varphi}_{1} \\
& =\lim _{P \rightarrow \infty}\left(-\frac{1}{2} \frac{M}{\Delta m}\right) \tilde{\varphi}_{1} .
\end{aligned}
$$

This relation means that for large $\boldsymbol{P}$ a constant proportionality between $\tilde{\varphi}_{1}$ and $\tilde{\varphi}_{2}$ can be established. We therefore can write relation $(2.28)$ in the equivalent form

$$
\tilde{\varphi}_{2}=-\frac{1}{2} \eta \tilde{\varphi}_{1}, \quad \text { for } P \rightarrow \infty,
$$

with $\eta:=M / \Delta m$.

By a similar but more extensive calculation it follows that

$\lim _{P \rightarrow \infty} M_{2}^{-1} V_{2} \tilde{\varphi}_{1}=\lim _{P \rightarrow \infty}\left(\frac{1}{P}\right) \hat{V}_{2} \tilde{\varphi}_{1}$

holds, where $\hat{V}$ remains finite for $P \rightarrow \infty$. We thus have $\lim _{P \rightarrow \infty} M_{2}^{-1} V_{2} \tilde{\varphi}_{1}=0$ and can immediately evaluate the limit of (2.12). This gives

$$
\tilde{\varphi}_{3}=\frac{1}{2} \eta^{2} \tilde{\varphi}_{1} \text { for } P \rightarrow \infty .
$$

Along similar lines the low energy limits $P \rightarrow 0$ for (2.11) and (2.12) can be discussed and the same procedures for the high energy limits as well as for the low energy limits can be applied to (2.24) and (2.25) for the left-hand solutions.

Summarizing the results of these procedures we eventually obtain

$\tilde{\varphi}_{2}=-\frac{1}{2} \eta \tilde{\varphi}_{1} ; \quad \tilde{\sigma}_{2}=-\frac{1}{2} \eta \tilde{\sigma}_{3}$

$\tilde{\varphi}_{3}=2\left(\frac{1}{2} \eta\right)^{2} \tilde{\varphi}_{1} ; \quad \tilde{\sigma}_{1}=2\left(\frac{1}{2} \eta\right)^{2} \tilde{\sigma}_{3}$, for $P \rightarrow \infty$,

and

$$
\begin{array}{ll}
\tilde{\varphi}_{2}=-\eta \tilde{\varphi}_{1} ; & \tilde{\sigma}_{2}=-\eta \tilde{\sigma}_{3} ; \\
\tilde{\varphi}_{3}=2(\eta)^{2} \tilde{\varphi}_{1} ; & \tilde{\sigma}_{1}=2(\eta)^{2} \tilde{\sigma}_{3},
\end{array}
$$

It is remarkable that the high energy limit can be obtained from the low energy limit by simply replacing $\eta$ by $1 / 2 \eta$.

For the futher evaluation of the wavefunctions their norms are needed. The norm is given by, cf. [7] and II,

$$
\langle b \quad b\rangle=\sum_{i k} \sigma^{i k} \varphi^{i k}=\sum_{i=1}^{3} \sigma_{i} \varphi_{i}=\sum_{i=1}^{3} \tilde{\sigma}_{i} \tilde{\varphi}_{i}
$$

i.e. the spinor expansion amplitudes of (2.10) and (2.23) can equivalently be used for the norm calculation provided the spinor basis is properly orthonormalized. Substitution of (2.32) into (2.34) yields

$$
\langle b \mid b\rangle=\frac{5}{4} \eta^{2} \tilde{\sigma}_{3} \tilde{\varphi}_{1} \text { for } P \rightarrow \infty .
$$

According to [7] we can approximately put $\tilde{\sigma}_{3}=\tilde{\varphi}_{1}^{+}$ and then obtain

$$
\langle b \mid b\rangle=\frac{5}{4} \eta^{2} \tilde{\varphi}_{1}^{+} \tilde{\varphi}_{1}=\left\langle\varphi_{1}^{n} \mid \varphi_{1}^{n}\right\rangle=1
$$

with $\varphi_{1}^{n}:=(2 / \sqrt{5}) \eta^{-1} \tilde{\varphi}_{1}$.

If now all these relations are substituted into (2.3) and the analogous relation for the left-hand solutions we have

$$
\varphi^{r r^{\prime}}=U^{r r^{\prime}} \varphi_{1}^{n} ; \quad \sigma^{r r^{\prime}}=S^{r r^{\prime}} \varphi_{1}^{n+}
$$

with

$$
\left(\begin{array}{l}
U^{11} \\
U^{21} \\
U^{12} \\
U^{22}
\end{array}\right)=\frac{2}{\sqrt{5}}\left(\begin{array}{lr}
\eta^{-1}-\frac{1}{2} \sqrt{2}+\frac{1}{2} \eta \\
\eta^{-1} & -\frac{1}{2} \eta \\
\eta^{-1} & -\frac{1}{2} \eta \\
\eta^{-1}+\frac{1}{2} \sqrt{2}+\frac{1}{2} \eta
\end{array}\right) \text { for } P \rightarrow \infty
$$

and

$$
\left(\begin{array}{l}
S^{11} \\
S^{21} \\
S^{12} \\
S^{22}
\end{array}\right)=\frac{2}{\sqrt{5}}\left(\begin{array}{lr}
\frac{1}{2} \eta-\frac{1}{2} \sqrt{2}+\eta^{-1} \\
\frac{1}{2} \eta & -\eta^{-1} \\
\frac{1}{2} \eta & -\eta^{-1} \\
\frac{1}{2} \eta+\frac{1}{2} \sqrt{2}+\eta^{-1}
\end{array}\right) \text { for } P \rightarrow \infty
$$

The corresponding expressions for the low energy limit $P \rightarrow 0$ can be obtained from (2.38) and (2.39) by replacing $\eta$ by $2 \eta$.

Finally, the wavefunction $\varphi_{1}^{n}$ has to be calculated. As $\varphi_{1}^{n}$ differs from $\tilde{\varphi}_{1}$ only by a constant normalization factor we can use (2.13) for the calculation of $\varphi_{1}^{n}$. In this case the application of limit procedures in analogy to (2.28) etc. is of no help for the solution of (2.13) since not a relation between wavefunctions is needed, but a wavefunction itself has to be derived. Nevertheless, a leading term approximation can be made in (2.13) which is in some respect similar to (2.28). A rough estimate shows that in such a leading term approximation (2.13) can be replaced by

$\left(2 Z_{2} M_{2}^{-1} Z_{2} M_{2}^{-1} Z_{2}+Z_{2} M_{2}^{-1} V_{2}\right) \varphi_{1}^{n}=0$ 
or equivalently by

$$
\left(2 Z_{2} M_{2}^{-1} Z_{2}+V_{2}\right) \varphi_{1}^{n}=0 .
$$

For brevity we do not explicitly discuss the approximate solution of (2.41). If all anisotropic parts of $V$ are neglected we obtain an approximate solution of (2.41) in the form

with

$$
\varphi_{1}^{n}\left(\boldsymbol{p}_{1}, \boldsymbol{p}_{2}\right)=\delta\left(\boldsymbol{P}-\boldsymbol{p}_{1}-\boldsymbol{p}_{2}\right) \chi(\boldsymbol{u} \mid \boldsymbol{P})
$$

$$
\chi(\boldsymbol{u} \boldsymbol{P})=Z_{2}(\boldsymbol{u}, \boldsymbol{P})^{-2} M_{2}(\boldsymbol{u}, \boldsymbol{P})^{2} c(\boldsymbol{P}),
$$

where $c(\boldsymbol{P})$ is a function which depends only on the center of mass momentum $\boldsymbol{P}$. For the derivation of (2.42), (2.43) it was assumed that an energy eigenvalue $E_{b}(\boldsymbol{P})=\left(m_{b}^{2}+\boldsymbol{P}^{2}\right)$ of $(2.41)$ does exist where $m_{b}$ is the preon-antipreon bound state rest mass. It was, however, not necessary to calculate this energy value explicitly.

According to (2.36) $\varphi_{1}^{n}$ has to be normalized to unity. This yields with (2.42) and (2.43)

$$
\begin{aligned}
\left\langle\varphi_{1}^{n} \mid \varphi_{1}^{n}\right\rangle & =\delta(0) \int \delta\left(\boldsymbol{P}-\boldsymbol{p}_{1}-\boldsymbol{p}_{2}\right)\left|\chi\left(\boldsymbol{p}_{1}-\boldsymbol{p}_{2} \mid \boldsymbol{P}\right)\right|^{2} \mathrm{~d} p_{1} \mathrm{~d} p_{2} \\
& =\delta(0) \int|\chi(\boldsymbol{u} \mid \boldsymbol{P})|^{2} \mathrm{~d} u=1
\end{aligned}
$$

if we assume that the bound state is characterized by fixed values of $s_{1}$ and $s_{2}$. The value $\delta(0)$ is the usual plane wave divergence which can be removed by suitably defined wave functions. This factor is of no interest here. The norm of $\chi$ reads explicitly

$$
\begin{aligned}
\chi \|= & \left.c(\boldsymbol{P})\right|^{2} \int\left[E_{b}(\boldsymbol{P})-E\left(\frac{1}{2}(\boldsymbol{P}+\boldsymbol{u})\right)-E\left(\frac{1}{2}(\boldsymbol{P}-\boldsymbol{u})\right)\right]^{-4} \\
& \cdot\left[E\left(\frac{1}{2}(\boldsymbol{P}+\boldsymbol{u})\right)^{-1}+E\left(\frac{1}{2}(\boldsymbol{P}-\boldsymbol{u})\right)^{-1}\right]^{4} \mathrm{~d} u .
\end{aligned}
$$

The integral (2.45) can be approximately evaluated in the high energy region with $P \gg M$ as well as in the low energy region with $P \ll M$.

In the high energy region polar coordinates are introduced and the radial integration over $u$ is split into two parts with $0 \leqq u \leqq P$ and $P \leqq u<\infty$. In doing so we obtain

$$
\|\chi\| \approx|c(\boldsymbol{P})|^{2} \mathscr{N} P^{-5}
$$

where $\mathscr{N}$ is numerical constant and $P:=\mid \boldsymbol{P}$.

In the low energy region we obtain by a similar evaluation method

$$
\left.\|\chi\| \approx c(\boldsymbol{P})\right|^{2} \cdot \mathcal{N}^{\prime} M^{-5}
$$

where $\mathcal{V}^{\prime}$ is a numerical constant.

Apart from $\mathcal{N}$ and $\mathscr{N}^{\prime}$ we can reduce (2.46) and (2.47) to a single expression by using the extra- polation

$$
\chi \| \approx|c(\boldsymbol{P})|^{2} \jmath^{\prime \prime} E(\boldsymbol{P})^{-5}
$$

which goes over into (2.46) or (2.47) respectively in the corresponding energy ranges. Then comparing (2.48) with (2.44) we obtain

$$
c(\boldsymbol{P}) \approx E(\boldsymbol{P})^{5 / 2}
$$

and the normalized wavefunction (2.43) reads

$$
\chi(\boldsymbol{u} \boldsymbol{P})=Z_{2}(\boldsymbol{u}, \boldsymbol{P})^{-2} M_{2}(\boldsymbol{u}, \boldsymbol{P})^{2} E(\boldsymbol{P})^{5 / 2} .
$$

For the evaluation of matrix elements it is sometimes convenient not to normalize the norm of $\chi$ but the integral over $\chi$ itself, i.e. we look for a function $\lambda(\boldsymbol{u}, \boldsymbol{P})=z \chi(\boldsymbol{u}, \boldsymbol{P})$ with

$$
\int \lambda(\boldsymbol{u}, \boldsymbol{P}) \mathrm{d} u=1 .
$$

By a calculation similar to those done above we obtain $z=E(\boldsymbol{P})^{-3 / 2}$ and thus

$$
\lambda(\boldsymbol{u}, \boldsymbol{P})=Z_{2}(\boldsymbol{u}, \boldsymbol{P})^{-2} M_{2}(\boldsymbol{u}, \boldsymbol{P})^{2} E(\boldsymbol{P})
$$

which again reproduces the results of the normalization (2.52) in the high energy as well as in the low energy region.

\section{High Momentum Fermion Wave Functions}

Due to the same reasoning as for boson wave functions, high momentum fermion wave functions are needed. The energy eigenvalue equation is for all composite particles the same and is given by (2.1). Apart from group-theoretical differences with respect to the determination of angular momentum quantum numbers the calculation of fermion states runs along the same lines as that for boson states. Since we do not undertake here to give an analysis of angular momentum determination we can take over the general line of calculation of Section 2.

The fermion states under consideration are assumed to be bound states of three preons and their general state functional can be written

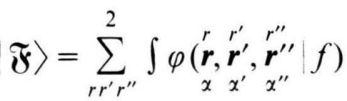

$$
\begin{aligned}
& \cdot j_{\alpha}^{r}(\boldsymbol{r}) j_{\alpha^{\prime}}^{r^{\prime}}\left(\boldsymbol{r}^{\prime}\right) j_{\alpha^{\prime \prime}}^{r^{\prime \prime}}\left(\boldsymbol{r}^{\prime \prime}\right)|0\rangle \mathrm{d} r \mathrm{~d} r^{\prime} \mathrm{d} r^{\prime \prime} .
\end{aligned}
$$

For the further evaluation the orthogonal transformation of the sources with respect to the auxiliary fields has to be performed, cf. Section 2. This 
transformation induces in the space of fermion wave functions an orthogonal transformation which formally reads

$$
\varphi^{i j k}=\sum_{\alpha \beta \gamma} U_{\alpha \beta \gamma}^{i j k} \varphi^{\alpha \beta \gamma} \quad\left(\begin{array}{l}
i, j, k=1,2 \\
x, \beta, \gamma=\varrho, \chi
\end{array}\right)
$$

and where only the auxiliary field indices are used while all other indices etc. are omitted for brevity. The transformation matrix $U$ of (3.2) was explicitly given in II and need not to be reproduced here.

The transformed wave functions themselves are not independent. Rather one has to employ antisymmetric combinations. This yields, cf. II, in the case of the three-preon bound state the following independent components

$$
\begin{aligned}
& \varphi_{1}:=\varphi^{\varrho \varrho \varrho}, \\
& \varphi_{2}:=a_{1}:=\frac{1}{3}\left(\varphi^{\varrho \varkappa \varrho}+\varphi^{\varrho \varrho \chi}+\varphi^{\chi \varrho \varrho}\right), \\
& \varphi_{3}:=a_{1}^{\prime}:=\frac{1}{3}\left(\varphi^{\varrho \chi \chi}+\varphi^{\chi \varrho \chi}+\varphi^{\chi \chi \varrho}\right), \\
& \varphi_{4}:=\varphi^{\varkappa \varkappa \% .}
\end{aligned}
$$

For these functions from (2.1) a set of four equations can be derived which were explicitly calculated by Hailer [97] and Lauxmann [99] and which can be written in the symbolical form

$\left(\begin{array}{cccc}3_{3} & \mathfrak{M}_{3} & 0 & 0 \\ \mathfrak{M}_{3}+\mathfrak{V}_{3} & 33_{3} & 2 \mathfrak{M}_{3} & 0 \\ \mathfrak{W}_{3} & 2 \mathfrak{M}_{3}+\mathfrak{B}_{3} & 3 \mathfrak{Z}_{3} & \mathfrak{M}_{3} \\ 0 & \mathfrak{W}_{3} & \mathfrak{M}_{3} & \mathfrak{3}_{3}\end{array}\right)\left(\begin{array}{c}\varphi_{1} \\ \varphi_{2} \\ \varphi_{3} \\ \varphi_{4}\end{array}\right)=0$

where the definitions of the symbols $3_{3}, \mathfrak{M}_{3}, \mathfrak{B}_{3}$, $\mathfrak{W}_{3}$ are given by these authors and need not be reproduced here.

The set of equations (3.4) can be resolved with respect to $\varphi_{2}, \varphi_{3}$ and $\varphi_{4}$ in terms of $\varphi_{1}$ by

$$
\begin{aligned}
\varphi_{2}= & -\mathfrak{M}_{3}^{-1} \mathfrak{3}_{3} \varphi_{1}=: \mathfrak{Q}_{1} \varphi_{1}, \\
\varphi_{3}= & \frac{1}{2}\left(3 \mathfrak{M}_{3}^{-1} 3_{3} \mathfrak{M}_{3}^{-1} \mathfrak{3}_{3}-1-\mathfrak{M}_{3}^{-1} \mathfrak{B}_{3}\right) \varphi_{1}=: \mathfrak{A}_{2} \varphi_{1}, \\
\varphi_{4}= & \left(-\frac{9}{2} \mathfrak{M}_{3}^{-1} \mathfrak{3}_{3} \mathfrak{M}_{3}^{-1} \mathfrak{3}_{3} \mathfrak{M}_{3}^{-1} \mathfrak{3}_{3}+\frac{7}{2} \mathfrak{M}_{3}^{-1} \mathfrak{3}_{3}\right. \\
& +\frac{3}{2} \mathfrak{M}_{3}^{-1} \mathfrak{3}_{3} \mathfrak{M}_{3}^{-1} \mathfrak{V}_{3}+\mathfrak{M}_{3}^{-1} \mathfrak{V}_{3} \mathfrak{M}_{3}^{-1} 3_{3} \\
& \left.-\mathfrak{M}_{3}^{-1} \mathfrak{W}_{3}\right) \varphi_{1}=: \mathfrak{A}_{3} \varphi_{1}
\end{aligned}
$$

and leads to an equation for $\varphi_{1}$ alone if (3.5), (3.6) and (3.7) are substituted in the last equation of (3.4). Formally this equation reads

$$
\left(\mathfrak{W}_{3} \mathfrak{H}_{1}+\mathfrak{M}_{3} \mathfrak{H}_{2}+3_{3} \mathfrak{I}_{3}\right) \varphi_{1}=0
$$

and can be used to calculate the energy eigenstates of the three preon system.

For a more detailed discussion of (3.5), (3.6), (3.7), and (3.8) it is necessary to expand $\varphi_{1}, \varphi_{2}, \varphi_{3}$ and $\varphi_{4}$ in terms of Dirac spinors. For the threepreon bound state such an expansion is given by

$$
\begin{gathered}
\varphi_{i}\left(\underset{\chi^{\prime}}{\boldsymbol{r}, \boldsymbol{r}^{\prime}, \boldsymbol{r}_{\alpha^{\prime \prime}}^{\prime \prime}} f\right)=\sum_{s s^{\prime} s^{\prime \prime}} \int \tilde{\varphi}_{i}\left(\boldsymbol{k} s, \boldsymbol{k}^{\prime} s^{\prime}, \boldsymbol{k}^{\prime \prime} s^{\prime \prime} \mid f\right) \\
\cdot u_{x}(\boldsymbol{k} s) u_{\alpha^{\prime}}\left(\boldsymbol{k}^{\prime} s^{\prime}\right) u_{\alpha^{\prime \prime}}\left(\boldsymbol{k}^{\prime \prime} s^{\prime \prime}\right) \\
\cdot e^{i \boldsymbol{k} \boldsymbol{r}+i \boldsymbol{k}^{\prime} \boldsymbol{r}^{\prime}+i \boldsymbol{k}^{\prime \prime} \boldsymbol{r}^{\prime \prime}} \mathrm{d} k \mathrm{~d} k^{\prime} \mathrm{d} k^{\prime \prime},
\end{gathered}
$$

$i=1,2,3,4$. The evaluation of (3.5), (3.6), (3.7), and (3.8) in terms of Dirac spinors according to the expansion (3.9) was performed by Hailer [97] and Lauxmann [99]. Thereby (3.5), (3.6), and (3.7) go over into a set of equations where the quantities $\left\{\varphi_{i}, \mathfrak{Z}_{3}, \mathfrak{M}_{3}, \mathfrak{B}_{3}, \mathfrak{M}_{3}\right\}$ are replaced by $\left\{\tilde{\varphi}_{i}, Z_{3}, M_{3}\right.$, $\left.V_{3}, W_{3}\right\}$ with

$Z_{3} \equiv Z\left(\boldsymbol{p}, \boldsymbol{p}^{\prime}, \boldsymbol{p}^{\prime \prime}\right):=\left[E-E(\boldsymbol{p})-E\left(\boldsymbol{p}^{\prime}\right)-E\left(\boldsymbol{p}^{\prime \prime}\right)\right]$,

where $E=\left[m_{\mathrm{f}}^{2}+\left(\boldsymbol{p}+\boldsymbol{p}^{\prime}+\boldsymbol{p}^{\prime \prime}\right)^{2}\right]^{1 / 2}, m_{\mathrm{f}} \equiv$ bound state fermion mass, and

$$
\begin{aligned}
M_{3} & \equiv M\left(\boldsymbol{p}, \boldsymbol{p}^{\prime}, \boldsymbol{p}^{\prime \prime}\right) \\
& :=-\Delta m M\left[E(\boldsymbol{p})^{-1}+E\left(\boldsymbol{p}^{\prime}\right)^{-1}+E\left(\boldsymbol{p}^{\prime \prime}\right)^{-1}\right] .
\end{aligned}
$$

The quantities $V_{3}$ and $W_{3}$ are integral kernels which for brevity are not explicitly given here.

For the complete description of the bound fermion states we also need the left-hand solutions of (2.1). For the fermion states under consideration these left-hand solutions have the general form

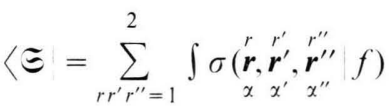

$$
\begin{aligned}
& \cdot\langle 0| \partial_{\alpha}^{r}(\boldsymbol{r}) \partial_{x^{\prime}}^{r^{\prime}}\left(\boldsymbol{r}^{\prime}\right) \partial_{\alpha^{\prime \prime}}^{r^{\prime \prime}}\left(\boldsymbol{r}^{\prime \prime}\right) \mathrm{d} r \mathrm{~d} r^{\prime} \mathrm{d} r^{\prime \prime} .
\end{aligned}
$$

The treatment of (3.12) runs along the same line as that of (3.1). In particular the set $\left\{\sigma^{i j k}\right\}$ is subjected to the same transformation (3.2) as the set $\left\{\varphi^{i j k}\right\}$. Applying the symmetry combinations to the transformed functions $\left\{\sigma^{\alpha \beta \gamma}\right\}$ we then obtain four independent functions $\left\{\sigma_{i}, i=1,2,3,4\right\}$ whose definition in terms of the set $\left\{\sigma^{\alpha \beta \gamma}\right\}$ is the same as given in (3.3) for the $\varphi$-functions. These functions are the left-hand solutions of (3.4). Resolution of these equations with respect to $\sigma_{1}, \sigma_{2}$ and $\sigma_{3}$ in terms of 
$\sigma_{4}$ yields

$$
\begin{aligned}
\sigma_{1} & =\sigma_{4}\left(-\frac{9}{2} 3_{3} \mathfrak{M}_{3}^{-1} 3_{3} \mathfrak{M}_{3}^{-1} 3_{3} \mathfrak{M}_{3}^{-1}+\frac{1}{2} 3_{3} \mathfrak{M}_{3}^{-1}\right. \\
& \left.\quad+3_{3} \mathfrak{M}_{3}^{-1} \mathfrak{W}_{3} \mathfrak{M}_{3}^{-1}+\mathfrak{M}_{3} \mathfrak{M}_{3}^{-1}\right) \\
\sigma_{2}= & \sigma_{4}\left(\frac{3}{2} 3_{3} \mathfrak{M}_{3}^{-1} 3_{3} \mathfrak{M}_{3}^{-1}+\frac{1}{2}\right) \\
\sigma_{3}= & -\sigma_{4} 3_{3} \mathfrak{M}_{3}^{-1}
\end{aligned}
$$

and leads to an equation for $\sigma_{4}$ alone which we suppress for brevity.

The expansion of the set $\left\{\sigma_{i}\right\}$ in terms of Dirac spinors is defined by

$$
\begin{aligned}
\sigma_{i}\left(\underset{x^{\prime}}{\boldsymbol{r}_{\chi^{\prime}}^{\prime}, \boldsymbol{r}_{\chi^{\prime \prime}}^{\prime \prime}} f\right) & =\sum_{s s^{\prime} s^{\prime \prime}} \int \tilde{\sigma}_{i}\left(\boldsymbol{k} s, \boldsymbol{k}^{\prime} s^{\prime}, \boldsymbol{k}^{\prime \prime} s^{\prime \prime} \mid f\right) \\
\cdot & u_{\alpha}^{+}(\boldsymbol{k} s) u_{\alpha^{\prime}}^{+}\left(\boldsymbol{k}^{\prime} s^{\prime}\right) u_{\alpha^{\prime \prime}}^{+}\left(\boldsymbol{k}^{\prime \prime} s^{\prime \prime}\right) \\
\cdot & e^{-i \boldsymbol{k} \boldsymbol{r}-i \boldsymbol{k}^{\prime} \boldsymbol{r}^{\prime}-i \boldsymbol{k}^{\prime \prime} \boldsymbol{r}^{\prime \prime}} \mathrm{d} k \mathrm{~d} k^{\prime} \mathrm{d} k^{\prime \prime}
\end{aligned}
$$

and the substitution of these expansions into (3.13), (3.14), and (3.15) as well as in the final equation for $\sigma_{4}$ leads to the replacement of $\left\{\sigma_{i}, \mathfrak{3}_{3}, \mathfrak{M}_{3}, \mathfrak{B}_{3}, \mathfrak{M}_{3}\right\}$ by $\left\{\tilde{\sigma}_{i}, Z_{3}, M_{3}, V_{3}, W_{3}\right\}$.

In order to obtain appropriate approximations for the equations (3.5), (3.6), (3.7), (3.13), (3.14), and (3.15) in analogy to the two-preon bound states, we introduce Jacobi coordinates for the three-preon states by

$$
\begin{aligned}
\boldsymbol{P} & =\boldsymbol{k}+\boldsymbol{k}^{\prime}+\boldsymbol{k}^{\prime \prime} ; & \boldsymbol{k} & =\frac{1}{3} \boldsymbol{P}-\frac{2}{3} \boldsymbol{q}+\boldsymbol{p} ; \\
\boldsymbol{q} & =\frac{1}{2}\left(\boldsymbol{k}-\boldsymbol{k}^{\prime}\right) ; & \boldsymbol{k}^{\prime} & =\frac{1}{3} \boldsymbol{P}+\frac{4}{3} \boldsymbol{q}-\frac{1}{2} \boldsymbol{p} ; \\
\boldsymbol{p} & =\frac{1}{3}\left[2 \boldsymbol{k}-\left(\boldsymbol{k}^{\prime}+\boldsymbol{k}^{\prime \prime}\right)\right] ; & \boldsymbol{k}^{\prime \prime} & =\frac{1}{3} \boldsymbol{P}-\frac{2}{3} \boldsymbol{q}-\frac{1}{2} \boldsymbol{p} ;
\end{aligned}
$$

Since we are merely interested in bound states we can assume that the functions of the sets $\left\{\tilde{\varphi}_{i}\right\}$ and $\left\{\tilde{\sigma}_{i}\right\}$ are sufficiently concentrated about the origin of relative momenta $\boldsymbol{q}$ and $\boldsymbol{p}$. Under this supposition we can perform limit procedures for large and small center of mass momenta $\boldsymbol{P}$ with respect to (3.5), (3.6), (3.7), (3.13), (3.14), (3.15). For (3.5) we obtain

$$
\begin{aligned}
\lim _{P \rightarrow \infty} \tilde{\varphi}_{2} & =-\lim _{P \rightarrow \infty} M_{3}^{-1} Z_{3} \tilde{\varphi}_{1} \\
& =\lim _{P \rightarrow \infty}\left(9 \Delta m M \frac{1}{P}\right)^{-1}\left(-\frac{1}{2} \frac{9 M^{2}}{P}\right) \tilde{\varphi}_{1} \\
& =\lim _{P \rightarrow \infty}\left(-\frac{1}{2} \frac{M}{\Delta m}\right) \tilde{\varphi}_{1}
\end{aligned}
$$

and

$$
\begin{aligned}
\lim _{P \rightarrow 0} \tilde{\varphi}_{2}- & \lim _{P \rightarrow 0} M_{3}^{-1} Z_{3} \tilde{\varphi}_{1} \\
& =\lim _{P \rightarrow 0}(3 \Delta m)^{-1}(-3 M) \tilde{\varphi}_{1} \\
& =\lim _{P \rightarrow 0}\left(-\frac{M}{\Delta m}\right) \tilde{\varphi}_{1} .
\end{aligned}
$$

Similar limit procedures can be performed for the other equations. In particular it turns out by detailed calculations that all terms containing $\mathfrak{B}_{3}$ or $\mathfrak{W}_{3}$ respectively do not contribute to the leading terms in the limit expressions and hence do not appear in these expressions.

Summarizing the results of these procedures we eventually get the relations

$$
\begin{array}{ll}
\tilde{\varphi}_{2}=-\frac{1}{2} \eta \tilde{\varphi}_{1} ; \quad \tilde{\sigma}_{3}=-\frac{1}{2} \eta \tilde{\sigma}_{4} ; \\
\tilde{\varphi}_{3}=\frac{3}{2}\left(\frac{1}{2} \eta\right)^{2} \tilde{\varphi}_{1} ; \quad \tilde{\sigma}_{2}=\frac{3}{2}\left(\frac{1}{2} \eta\right)^{2} \tilde{\sigma}_{4} ; \quad \text { for } P \rightarrow \infty, \\
\tilde{\varphi}_{4}=-\frac{9}{2}\left(\frac{1}{2} \eta\right)^{3} \tilde{\varphi}_{1} ; \quad \tilde{\sigma}_{1}=-\frac{9}{2}\left(\frac{1}{2} \eta\right)^{3} \tilde{\sigma}_{4} ;
\end{array}
$$

and

$\tilde{\varphi}_{2}=-\eta \tilde{\varphi}_{1} ; \quad \tilde{\sigma}_{3}=-\eta \tilde{\sigma}_{4} ;$

$\tilde{\varphi}_{3}=\frac{3}{2} \eta^{2} \tilde{\varphi}_{1} ; \quad \tilde{\sigma}_{2}=\frac{3}{2} \eta^{2} \tilde{\sigma}_{4} ;$ for $P \rightarrow 0$.

$\tilde{\varphi}_{4}=-\frac{9}{2} \eta^{2} \tilde{\varphi}_{1} ; \quad \tilde{\sigma}_{1}=-\frac{9}{2} \eta^{3} \tilde{\sigma}_{4} ;$

The high energy limit can be obtained from the low energy limit by simply replacing $\eta$ by $\frac{1}{2} \eta$.

The norm of the three-preon states is given by, cf. II,

$\langle f f\rangle=\sum_{i j k} \sigma^{i j k} \varphi^{i j k}=\sum_{i=1}^{4} \sigma_{i} \varphi_{i}=\sum_{i=1}^{4} \tilde{\sigma}_{i} \tilde{\varphi}_{i}$.

Substitution of (3.19) yields for $\eta<0$

$$
\langle f \mid f\rangle=-\frac{3}{2} \eta^{3} \tilde{\sigma}_{4} \tilde{\varphi}_{1}=\frac{3}{2} \mid \eta^{3} \tilde{\varphi}_{1}^{+} \tilde{\varphi}_{1}
$$

if according to [7] $\tilde{\sigma}_{4}$ is replaced by $\tilde{\varphi}_{1}^{+}$. Substitution of $\tilde{\varphi}_{1}=(2 / 3)^{1 / 2} \mid \eta^{-3 / 2} \varphi_{1}^{n}$ with $\varphi_{1}^{n}=1$ leads to

$$
\langle f \mid f\rangle=\left\langle\varphi_{1}^{n} \varphi_{1}^{n}\right\rangle=1
$$

apart from the plane wave normalization factor $\delta(0)$ which is of no interest here. If now all these relations are substituted into (3.2) and the analogous equation for the left-hand solutions, this gives

$$
\varphi^{r r^{\prime} r^{\prime \prime}}=U^{r r^{\prime} r^{\prime \prime}} \varphi_{1}^{n} ; \quad \sigma^{r r^{\prime} r^{\prime \prime}}=S^{r r^{\prime} r^{\prime \prime}} \varphi_{1}^{n+} .
$$

Apart from numerical factors $U$ and $S$ are explicitly written out in II for the low energy limit $P \rightarrow 0$. The 
high energy limit $P \rightarrow \infty$ results from these formulas by the replacement $\eta \rightarrow \frac{1}{2} \eta$. For brevity we refer to II and do not reproduce these formulas.

Finally, the wave function $\varphi_{1}^{n}$ has to be calculated. A direct calculation by means of the wave equation (3.8) for $\varphi_{1}^{n}$ is in preparation. As long as no results of this calculation are available we have to make a plausible guess. Such a guess was made in II by assuming the three-preon bound state to be a product of two internal wave functions of bound boson states in the Jacobi coordinates. In the case of a representation by means of Dirac spinors (3.9) for $\varphi_{1}^{n}$ the corresponding decomposition reads

$$
\begin{aligned}
& \varphi_{1}^{n}\left(\boldsymbol{k} s, \boldsymbol{k}^{\prime} s^{\prime}, \boldsymbol{k}^{\prime \prime} s^{\prime \prime} \boldsymbol{P}\right) \\
& \quad=\delta\left(\boldsymbol{P}-\boldsymbol{k}-\boldsymbol{k}^{\prime}-\boldsymbol{k}^{\prime \prime}\right) \chi(\boldsymbol{q} \boldsymbol{P}) \chi(\boldsymbol{p} P),
\end{aligned}
$$

where $\boldsymbol{P}$ is the center of mass momentum and $\chi$ is defined by (2.50) and where it is assumed that the quantum numbers $s, s^{\prime}, s^{\prime \prime}$ have fixed values which are suppressed on the right-hand side of (3.25). Apart from the plane wave normalization factor $\delta(0)$ the function $\varphi_{1}^{n}$ defined by (3.25) is automatically normalized to unity as the constituent functions $\chi$ are normalized to unity.

\section{Quantization Terms and Formfactors}

Having prepared the bound state wave functions of composite bosons and fermions in such a way that they are valid in the low energy as well as in the high energy range we can now study the weak mapping of a nonlinear spinorfield onto a fermionboson coupling theory in the high energy limit.

The nonlinear spinorfield model is defined by the field equations with scalar interaction, cf. I,

$$
\begin{aligned}
{\left[\left(-i \gamma^{\mu} \partial_{\mu}+m_{1}\right)\right.} & \left.\left(-i \gamma^{\varrho} \partial_{\varrho}+m_{2}\right)\right]_{\alpha \beta} \psi_{\beta}(x) \\
& =g V_{\alpha \beta \gamma \delta} \psi_{\beta}(x) \bar{\psi}_{\gamma}(x) \psi_{\delta}(x)
\end{aligned}
$$

which exhibit self-regularization, relativistic invariance and locality for canonical quantization at the quantum level.

It was proved by the author [3] and by Grosser [4] that the set of nonlinear equations $r=1,2$

$$
\begin{aligned}
& \left(-i \gamma^{\mu} \partial_{\mu}+m_{r}^{2}\right)_{x \beta} \varphi_{\beta}^{r}(x) \\
& \quad=g \lambda_{r} \sum_{s t u=1} V_{x \beta \gamma \delta} \varphi_{\beta}^{s}(x) \bar{\varphi}_{\gamma}^{t}(x) \varphi_{\delta}^{u}(x)
\end{aligned}
$$

is connected with (4.1) by a biunique map where this map is defined by the compatible relations

$$
\begin{aligned}
& \psi_{x}(x)=\varphi_{x}^{1}(x)+\varphi_{\alpha}^{2}(x), \\
& \varphi_{x}^{1}(x)=\lambda_{1}\left(-i \gamma^{\mu} \partial_{\mu}+m_{2}\right)_{\alpha \beta} \psi_{\beta}(x), \\
& \varphi_{x}^{2}(x)=\lambda_{2}\left(-i \gamma^{\mu} \partial_{\mu}+m_{1}\right)_{\alpha \beta} \psi_{\beta}(x)
\end{aligned}
$$

with $\lambda_{1}:=-(\Delta m)^{-1} 1 / 2, \quad \lambda_{2}:=(\Delta m)^{-1} 1 / 2$ and $\Delta m$ defined by (2.17).

Furthermore, it has been convenient to introduce the transformed field quantities

$$
\Phi^{1}(x):=\lambda_{1}^{-1 / 2} \varphi^{1}(x) ; \quad \Phi^{2}(x):=\left|\lambda_{2}\right|^{-1 / 2} \varphi^{2}(x) .
$$

The quantization of this model was performed in [7] and of a similar, more complicated model in [6]. The quantum states of this model are described by state functionals $\mathfrak{I}[j, a]\rangle$ with respect to the state a) where $j \equiv j_{x}^{r}(x)$ are sources with superspinor indices $\alpha$. For concrete calculations it is necessary to introduce normal transforms by

$$
\mathfrak{I}\rangle=Z_{0}[j]|\mathfrak{F}\rangle
$$

with

$$
Z_{0}[j]:=\exp \left[\sum_{r r^{\prime}} \int_{j} j_{x}^{r}(x) F_{\alpha \beta}^{r r^{\prime}}\left(x, x^{\prime}\right) j_{\beta}{ }^{r^{\prime}}\left(x^{\prime}\right) \mathrm{d}^{4} x \mathrm{~d}^{4} x^{\prime}\right],
$$

where the $F$-functions in superspinor indices are the free field propagators of (4.2).

The energy representation of the spinorfield, i.e. the corresponding functional equation for functional energy eigenstates of this field was discussed in [6] and for the above case given in [7]. It reads

$$
\begin{aligned}
E|\mathfrak{\mho}\rangle= & \sum_{r=1}^{2} \int j_{\varepsilon}^{r}(\boldsymbol{r}) G_{\varepsilon \alpha}^{0}\left(i \boldsymbol{G}_{\alpha \beta} \cdot \nabla-m_{r} \delta_{\alpha \beta}\right) \partial_{\beta}^{r}(\boldsymbol{r}) \mathrm{d} r|\mathfrak{F}\rangle \\
& +g \int\left[j_{\varepsilon}^{1}(\boldsymbol{r})-j_{\varepsilon}^{2}(\boldsymbol{r})\right] G_{\varepsilon \alpha}^{0} V_{\alpha \beta \gamma \delta}^{a} \\
& \cdot \mathrm{d}_{\beta}(\boldsymbol{r}) \mathrm{d}_{\gamma}(\boldsymbol{r}) \mathrm{d}_{\delta}(\boldsymbol{r}) \mathrm{d} r|\mathfrak{F}\rangle
\end{aligned}
$$

with

$$
\mathrm{d}_{\beta}(\boldsymbol{r}):=\sum_{r=1}^{2}\left[\partial_{\beta}^{r}(\boldsymbol{r})-\int F_{\beta \beta^{\prime}}^{r r^{\prime}}\left(\boldsymbol{r}, \boldsymbol{r}^{\prime}\right) j_{\beta^{\prime}}^{r^{\prime}}\left(\boldsymbol{r}^{\prime}\right) \mathrm{d} r^{\prime}\right] .
$$

For details of the definition of all quantities in the above expression we refer to [6], [7] and preceding papers cited there. In particular, $\lambda_{1}$ is absorbed in $g$. Furthermore, the bound state equation (2.1) is the diagonal part of (4.7) with respect to the state functionals $|\mathfrak{\mho}\rangle$. 
In order to avoid lengthy expressions, in I a more compact notation was introduced by replacing $j_{\alpha}^{r}(\boldsymbol{r})$ by $j_{x}$ etc. With this notation in I the weak mapping principle was applied to (4.7) by the introduction of the following representation for the state functional $\mathfrak{\mho}\rangle$ with $|\mathfrak{F}[j]\rangle \equiv|\mathfrak{F}[b, l, \bar{l}]\rangle$ and

$$
\begin{array}{r}
|\mathfrak{F}\rangle=\sum_{N K L} c\left(n_{1} \ldots n_{N}, q_{1} \ldots q_{K}, w_{1} \ldots w_{L}\right) \\
\cdot b_{n_{1}} \ldots b_{n_{N}} l_{q_{1}} \ldots l_{q_{K}} \bar{l}_{w_{1}} \ldots \bar{l}_{w_{L}}|0\rangle .
\end{array}
$$

If the spinorfield sources $j_{\alpha}$ are decomposed into spinor parts $j_{u}$ and conjugate spinor parts $\overline{j_{u}}$, the bound state source operators $b_{n}, l_{q}, \bar{l}_{w}$ are expressed by the definitions

$$
\begin{aligned}
& b_{n}:=C_{n}^{u r} j_{u} \overline{j_{r}}, \\
& l_{q}:=C_{q}^{r s t} j_{r} j_{s} j_{t}, \\
& \bar{l}_{w}:=\bar{C}_{w}^{a b c} \overline{j_{a}} \overline{j_{b}} \overline{j_{c}},
\end{aligned}
$$

where $\left\{C_{n}^{u v}\right\}$ and $\left\{C_{q}^{r s t}\right\}$ etc. are boson or fermion wave functions respectively, cf. I.

The representation of the functional energy states $|\mathfrak{F}\rangle$ in terms of bound state source operators as given by (4.9) induces a transformation of the corresponding functional equation (4.7). If this equation is written in the compact form $E|\mathfrak{F}\rangle=\mathscr{H}|\mathfrak{F}\rangle$ as a result of (4.9), (4.10), (4.11), (4.12) we have

$$
\mathscr{H}[j, \bar{j}, \partial, \bar{\partial}] \equiv \mathscr{H}\left[b, l, \bar{l}, \frac{\delta}{\delta b}, \frac{\delta}{\delta l}, \frac{\delta}{\delta \bar{l}}\right] .
$$

In I, II, III the bound state representation of $\mathscr{H}$, i.e. the right-hand side expression of (4.13) was extensively studied for the case of low energy processes. It turned out that in this range the bound state representation of $\mathscr{H}$ leads to a hierarchy of interactions where only the leading terms are of interest since the higher order terms have only a negligible influence on the physical reactions. A closer inspection of the results obtained so far reveals that this hierarchy of interactions is generated by the normalization properties and the number of participating wave functions in the various interaction terms. This situation is not changed qualitatively by the transition to the high energy range. We thus assume:

The hierarchy of interactions in the bound state representation of $\mathscr{H}$ is not qualitatively changed by the transition from the low energy range to the high energy range.
This assumption facilitates the subsequent calculations considerably, since we can concentrate upon the essential terms and can omit the lengthy calculations of the other negligible terms. In the following we take this assumption for granted and apply it. According to II and III in this case the functional energy operator $\mathscr{H}$ of (4.13) is given by the expression

$$
\mathscr{H}=\mathscr{H}_{b b}^{0}+\mathscr{H}_{l l}^{0}+\mathscr{H}_{l l}^{0}+\mathscr{H}_{b l}^{1}+\mathscr{H}_{b l}^{1}+\mathscr{H}_{l l}^{1}
$$

with

$$
\begin{aligned}
& \mathscr{H}_{b b}^{0}:=\left(\sum_{n} E_{n}^{b} b_{n} \frac{\delta}{\delta b_{n}}\right)^{1}, \\
& \mathscr{H}_{l l}^{0}:=\left(\sum_{q} E_{q}^{f} l_{q} \frac{\delta}{\delta l_{q}}\right)^{\mathrm{I}}, \\
& \mathscr{H}_{b l}^{1}:=\left(\sum_{n q q^{\prime}} V_{x \beta \gamma \delta}^{a} 6 C_{q}^{\beta \delta u} C_{n}^{v \gamma} R_{\text {xul }}^{q^{\prime}} l_{q^{\prime}} \frac{\delta}{\delta l_{q}} \frac{\delta}{\delta b_{n}}\right)^{\mathrm{I}},
\end{aligned}
$$

$$
\begin{aligned}
\mathscr{P}_{\mid \bar{T}}^{1}:=\left(-\sum_{\substack{q q^{\prime} \\
w w^{\prime}}} V_{\alpha \beta \gamma \delta}^{a} F_{\beta \beta^{\prime}} 3 C_{q}^{\delta s t} 3 \bar{C}_{w}^{\gamma u r} R_{x s t}^{q^{\prime}}\right. \\
\left.\cdot \bar{R}_{\beta^{\prime} u r}^{w^{\prime}} l_{q^{\prime}} \dot{\bar{l}}_{w^{\prime}} \frac{\delta}{\delta \bar{l}_{w}} \frac{\delta}{\delta l_{q}}\right)^{\mathrm{I}},
\end{aligned}
$$

where the superscript I means that in all expressions only bound state contributions are taken into account. The operators $\mathscr{H}_{\bar{I}}^{0}$ and $\mathscr{H}_{b \bar{l}}^{1}$ are obtained from (4.16) and (4.17) by replacing all fermion parts by antifermion parts. Further evaluations can be found in II and III.

We now evaluate (4.14) by means of the improved wave functions derived in Sects. 2 and 3. As (4.15) and (4.16) are the boson and fermion kinetic energies which result from the inclusion of (2.1) in the transformation of the complete $\mathscr{H}$, cf. II, we need to evaluate only the terms (4.17) and (4.18). With slight modifications this evaluation runs along the same lines as that done in III. So many calculations can be taken over from III. We therefore give the results of these calculations directly and dicuss only deviations from the procedure performed in III.

We first consider (4.17). Collecting the relations (2.37), (2.38), (2.42), and (2.50) for boson states and the relations (3.24) and (3.25) for fermion states and taking over from II the definitions of the dual states we substitute these expressions into (4.17). Due to the wave function representations (2.10) and (3.9) 
we have to evaluate (4.17) in momentum space in contrast to III, where this expression was evaluated in coordinate space. A straightforward calculation then yields

$$
\begin{gathered}
\mathscr{H}_{b l}^{1}=\sum_{t t^{\prime}} \eta^{-2} \int F\left(\boldsymbol{k}, \boldsymbol{q} t, \boldsymbol{q}^{\prime} t^{\prime}\right) \delta\left(\boldsymbol{k}+\boldsymbol{q}-\boldsymbol{q}^{\prime}\right) \\
\cdot\left[\frac{\delta}{\delta b_{+k}}+\frac{\delta}{\delta b_{-k}}\right] l_{t \boldsymbol{q}} \frac{\delta}{\delta l_{t^{\prime} \boldsymbol{q}^{\prime}}} \mathrm{d} k \mathrm{~d} q \mathrm{~d} q^{\prime} .
\end{gathered}
$$

Owing to the representations (2.10) and (3.9) the general formfactor $F\left(\boldsymbol{k}, \boldsymbol{q} t, \boldsymbol{q}^{\prime} t^{\prime}\right)$ contains a spindependent part and a scalar part. The spin-dependent part is a function bounded everywhere in the whole range of all variables which occur in this part. In order to simplify the expression for $F$ it is thus justified to approximate the spin-dependent part by neglecting all internal variables of this part. In this case the spin-dependent part only depends on the remaining center of mass momenta of the various participating composite particles and $F$ can be factorized in the form

$F\left(\boldsymbol{k}, \boldsymbol{q} t, \boldsymbol{q}^{\prime} t^{\prime}\right):=K_{\mathrm{s}}\left(\boldsymbol{k}, \boldsymbol{q} t, \boldsymbol{q}^{\prime} t^{\prime}\right) f\left(\boldsymbol{k}, \boldsymbol{q}, \boldsymbol{q}^{\prime}\right)$,

where the spin-dependent part $K_{\mathrm{s}}$ is defined by

$K_{\mathrm{s}}\left(\boldsymbol{k}, \boldsymbol{q} t, \boldsymbol{q}^{\prime} t^{\prime}\right):=V_{\chi \beta \gamma \delta}^{a} \chi_{x^{\prime} \gamma}(\boldsymbol{k}) \chi_{\beta \delta x}^{t^{\prime}}\left(\boldsymbol{q}^{\prime}\right) \chi_{\chi \varkappa \varkappa^{\prime}}^{t}(\boldsymbol{q})$

with

$\chi_{x^{\prime} \gamma}(\boldsymbol{k}):=u_{\varkappa^{\prime}}\left(\frac{1}{2} \boldsymbol{k}, s_{1}\right) v_{\gamma}^{+}\left(\frac{1}{2} \boldsymbol{k}, s_{2}\right)$,

$\chi_{\beta \delta}^{\prime \prime}\left(\boldsymbol{q}^{\prime}\right):=u_{\beta}\left(\frac{1}{3} \boldsymbol{q}^{\prime}, s_{1}^{\prime}\right) u_{\delta}\left(\frac{1}{3} \boldsymbol{q}^{\prime}, s_{2}^{\prime}\right) u_{\varkappa}\left(\frac{1}{3} \boldsymbol{q}^{\prime}, s_{3}^{\prime}\right) \quad$ etc.

while the scalar part $f$ is defined by

$$
\begin{aligned}
& f\left(\boldsymbol{k}, \boldsymbol{q}, \boldsymbol{q}^{\prime}\right):=\int \chi(\boldsymbol{s} \boldsymbol{k}) \chi\left(\boldsymbol{t} \boldsymbol{q}^{\prime}\right) \chi\left(\boldsymbol{u} \mid \boldsymbol{q}^{\prime}\right) \\
& \cdot \chi\left(-\frac{1}{3} \boldsymbol{q}+\frac{1}{3} \boldsymbol{q}^{\prime}-\boldsymbol{t}-\frac{1}{2} \boldsymbol{u} \boldsymbol{q}\right) \\
& \cdot \chi\left(-\frac{1}{2} \boldsymbol{k}-\frac{1}{3} \boldsymbol{q}^{\prime}+\frac{2}{3} \boldsymbol{q}+\boldsymbol{s}+\boldsymbol{t}+\frac{1}{2} \boldsymbol{u} \boldsymbol{q}\right) \mathrm{d} s \mathrm{~d} t \mathrm{~d} u .
\end{aligned}
$$

According to the approximate calculation of the wave functions (2.10) and (3.9) their constituents are assumed to have fixed spin quantum numbers $s$, which in turn lead to fixed spin quantum numbers of the corresponding composite particles. As the bosons are assumed to have spin zero, only the spin quantum numbers $t$ and $t^{\prime}$ of the composite fermions then appear in the final formula (4.21) of the spin-dependent part of the formfactor.

The calculation of the term $\mathscr{H}_{b} L$ is completely analogous to the procedure described above.
Finally, we have to calculate (4.18). This calculation again runs along the same lines as in III. It is, however, a little bit more complicated. In particular the lemma about the relation between the product of the fermion-antifermion source operators $l_{\rho}(\boldsymbol{r})$. $\bar{l}_{Q^{\prime}}\left(\boldsymbol{r}^{\prime}\right)$ and the boson source operators $b_{\mathrm{s}}(\boldsymbol{k})$ plays a central role in this calculation. In III this lemma was proved with respect to the special wave functions for composite fermions and bosons derived in II. So we have to repeat this proof by using the improved wave functions given in Sect. 2 and 3. For brevity we give only the result of this procedure. In III the relation was derived

$b_{\mathrm{s} k}=\sum_{b b^{\prime}} C_{\mathrm{s} k}^{\alpha \beta} F^{\alpha^{\prime} \beta^{\prime}} F^{\alpha^{\prime \prime} \beta^{\prime \prime}} R_{\alpha \alpha^{\prime} \alpha^{\prime \prime}}^{q} \bar{R}_{\beta \beta^{\prime} \beta^{\prime \prime}}^{q^{\prime}} l_{q} \bar{l}_{q^{\prime}}$

which is the starting point of our modified calculation. If we employ the improved wave functions of Sect. 2 and 3 and observe the definition of dual states, then the substitution of these quantities into (4.23) and subsequent calculation yields

$b_{\mathrm{S}}(\boldsymbol{k})=\int e^{-i \boldsymbol{k}\left(\boldsymbol{r}+\boldsymbol{r}^{\prime}\right) 1 / 2} \xi_{\alpha \beta}^{\mathrm{s}}\left(\boldsymbol{r}-\boldsymbol{r}^{\prime} \boldsymbol{k}\right) l_{\alpha}(\boldsymbol{r}) \bar{l}_{\alpha^{\prime}}\left(\boldsymbol{r}^{\prime}\right) \mathrm{d} r \mathrm{~d} r^{\prime}$

with

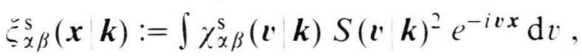

where the overlap factor $S^{\prime}$ is defined by

$S(\boldsymbol{v} \boldsymbol{k}):=\int \chi\left(\boldsymbol{u} \frac{1}{2}(\boldsymbol{k}+\boldsymbol{v})\right) \chi\left(\boldsymbol{u} \frac{1}{2}(\boldsymbol{k}-\boldsymbol{v})\right) \mathrm{d} u$.

In particular we have $S(0 \boldsymbol{k}) \equiv \| \chi=1$. Hence if we assume that the boson wave function $\chi(\boldsymbol{v} k)$ is sufficiently localized in $v$ about the origin we have $\xi^{\mathrm{s}} \approx \chi$ and in this approximation our result coincides with that of III. In the following we will apply this assumption. Then (4.24) can be inverted to give

$$
\begin{aligned}
l_{\varrho}(\boldsymbol{q}) & \bar{l}_{\varrho^{\prime}}(\boldsymbol{k}) \\
& =\chi_{\varrho \varrho^{\prime}}\left(-\frac{1}{2}(\boldsymbol{q}+\boldsymbol{k}) \mid \boldsymbol{k}-\boldsymbol{q}\right)(-1)^{\mathrm{s}} b_{\mathrm{s}}(\boldsymbol{k}-\boldsymbol{q})
\end{aligned}
$$

in momentum space. Having derived this auxiliary formula we now proceed analogously to the treatment of (4.17). We use the relations (3.24) and (3.25) for fermion states and take over the definitions of the corresponding dual states from II. Then we substitute these expressions in (4.18). Due to the wave function representation (3.9) we have to evaluate (4.18) in momentum space in contrast to III, where this expression was evaluated in coordinate space. If we further take into account (4.27) 
then a straight-forward calculation yields

$$
\begin{aligned}
\mathscr{H}_{i} t=\sum_{t t^{\prime}} \eta^{-2} \int \hat{F}\left(\boldsymbol{k}, \boldsymbol{q} t, \boldsymbol{q}^{\prime} t^{\prime}\right) \delta\left(\boldsymbol{k}+\boldsymbol{q}-\boldsymbol{q}^{\prime}\right) \\
\cdot\left[b_{+\boldsymbol{k}}-b_{-\boldsymbol{k}}\right] \frac{\delta}{\delta \bar{l}_{t \boldsymbol{q}}} \frac{\delta}{\delta l_{t^{\prime} \boldsymbol{q}^{\prime}}} \mathrm{d} k \mathrm{~d} q \mathrm{~d} q^{\prime} .
\end{aligned}
$$

With respect to the formfactor $\hat{F}\left(\boldsymbol{k}, \boldsymbol{q} t, \boldsymbol{q}^{\prime} t^{\prime}\right)$, however, we cannot obtain such a simple approximation (4.20) as is valid for $F$. Rather the spin parts and the orbital parts are correlated. The corresponding formula is given by

$$
\begin{aligned}
\hat{F} & \left(\boldsymbol{k}, \boldsymbol{q} t, \boldsymbol{q}^{\prime} t^{\prime}\right):=\int K^{t t^{\prime}}\left(\boldsymbol{q}, \boldsymbol{q}^{\prime}, \frac{1}{2}(\boldsymbol{v}-\boldsymbol{k}), \frac{1}{2}(\boldsymbol{v}+\boldsymbol{k})\right) \\
& \cdot \chi(\boldsymbol{s}, \boldsymbol{u} \boldsymbol{q}) \bar{\chi}\left(\boldsymbol{s}^{\prime}, \boldsymbol{u}^{\prime} \mid \boldsymbol{q}^{\prime}\right) \\
& \cdot \chi\left(-\boldsymbol{s}, \frac{2}{3} \boldsymbol{q}-\boldsymbol{u}-\frac{2}{3}\left(\boldsymbol{v}-\frac{1}{2} \boldsymbol{k}\right) \mid \boldsymbol{v}-\frac{1}{2} \boldsymbol{k}\right) \\
& \cdot \bar{\gamma}\left(-\boldsymbol{s}^{\prime}, \frac{2}{3} \boldsymbol{q}^{\prime}-\boldsymbol{u}^{\prime}-\frac{2}{3}\left(\boldsymbol{v}+\frac{1}{2} \boldsymbol{k}\right) \mid \boldsymbol{v}+\frac{1}{2} \boldsymbol{k}\right) \\
& \cdot \chi(-\boldsymbol{v} \mid \boldsymbol{k}) \mathrm{d} t \mathrm{~d} s \mathrm{~d} s^{\prime} \mathrm{d} u \mathrm{~d} u^{\prime}
\end{aligned}
$$

with the spin part

$$
\begin{aligned}
& K^{t t^{\prime}}\left(\boldsymbol{q}, \boldsymbol{q}^{\prime}, \boldsymbol{k}, \boldsymbol{k}^{\prime}\right):=\sum_{s s^{\prime}} V_{x \beta \gamma \delta}^{a} \chi_{\beta \lambda \lambda \lambda^{\prime}}^{t}(\boldsymbol{q}) \chi_{\gamma \varkappa x^{\prime}}^{t^{\prime}}\left(\boldsymbol{q}^{\prime}\right)^{+} \\
& \cdot \chi_{\chi \lambda \lambda \lambda^{\prime}}^{\mathrm{s}}(\boldsymbol{k}) \chi_{\delta \varkappa x^{\prime}}^{s^{\prime}}\left(\boldsymbol{k}^{\prime}\right)^{+} \psi_{\varrho}^{s}(\boldsymbol{k})^{+} \psi_{\varrho^{\prime}}^{s^{\prime}}\left(\boldsymbol{k}^{\prime}\right) u_{\varrho}(\boldsymbol{k}) v_{\varrho^{\prime}}^{+}\left(\boldsymbol{k}^{\prime}\right)
\end{aligned}
$$

and with the abbreviation

$$
\chi(\boldsymbol{s}, \boldsymbol{u} \mid \boldsymbol{q}):=\chi(\boldsymbol{s} \mid \boldsymbol{q}) \chi(\boldsymbol{u} \mid \boldsymbol{q}) .
$$

In order to compare the results of the weak mapping of the nonlinear spinorfield (4.1) defined by the state expansion (4.9) with the functional energy representation of a phenomenological bosonfermion coupling theory the final step needed is the transition to normal transforms of composite particles. According to III we define this normal transform by the relation $|\mathfrak{F}\rangle=Z_{1}[j]|\mathfrak{5}\rangle$ with

$$
\begin{aligned}
Z_{1}[j]:=\exp \left[-\frac{1}{2} j_{\alpha} \bar{j}_{\beta} F_{\alpha \beta, \alpha^{\prime} \beta^{\prime}}^{4} j_{\alpha^{\prime}} \bar{j}_{\beta^{\prime}}\right. \\
\\
\left.\quad-j_{\alpha} j_{\beta} j_{\gamma} F_{\alpha \beta \gamma, \alpha^{\prime} \beta^{\prime} \gamma^{\prime}}^{6} \overline{j_{\alpha^{\prime}}} \overline{j_{\beta^{\prime}}} \bar{j}_{\gamma^{\prime}}\right]
\end{aligned}
$$

and with

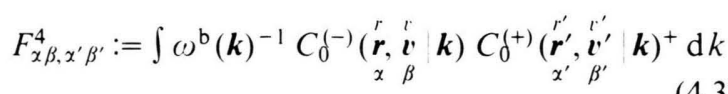

and

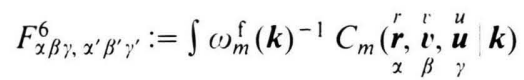

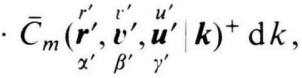

where $\left\{C_{\delta}^{ \pm}(\boldsymbol{k}), C_{m}(\boldsymbol{k}), \bar{C}_{m}(\boldsymbol{k})\right\}$ are the corresponding boson and fermion bound state functions.

The application of the weak mapping to (4.31) yields

$$
\begin{aligned}
Z_{1}[j] & \equiv Z_{1}[b, l, \bar{l}] \\
& =\exp \left[-\frac{1}{2} \sum_{n n^{\prime}} b_{n} \Delta_{n n^{\prime}} b_{n^{\prime}}-\sum_{q w} l_{q} S_{q w} \bar{l}_{w}\right]
\end{aligned}
$$

with

$\Delta_{n n^{\prime}}:=\delta_{n 1} \delta\left(\boldsymbol{q}-\boldsymbol{q}^{\prime}\right) \omega_{n}^{\mathrm{b}}(\boldsymbol{q})^{-1} \delta_{n^{\prime} 2}=: \delta\left(\boldsymbol{q}-\boldsymbol{q}^{\prime}\right) \Delta(\boldsymbol{q})$,

$S_{q w}:=\delta_{n n^{\prime}} \delta\left(\boldsymbol{q}-\boldsymbol{q}^{\prime}\right) \omega_{n}^{\mathrm{f}}(\boldsymbol{q})^{-1}=: \delta\left(\boldsymbol{q}-\boldsymbol{q}^{\prime}\right) S(\boldsymbol{q})$,

i.e. (4.35) equals the Fouriertransform of (1.12). Then we have

$$
\begin{aligned}
\mathfrak{F}\rangle=\exp \left[-\frac{1}{2} \int b_{-\boldsymbol{k}} \Delta(\boldsymbol{k}) b_{+\boldsymbol{k}} \mathrm{d} k\right. \\
\left.-\int l_{\boldsymbol{l} \boldsymbol{q}} S(\boldsymbol{q}) \bar{l}_{t \boldsymbol{q}} \mathrm{d} q\right]|\mathfrak{(}\rangle
\end{aligned}
$$

and this transformation is to be applied to the initial equation (4.7) in the leading term approximation (4.14). Collecting the results of all calculations in this section this equation reads

$$
\begin{aligned}
E|\mathfrak{F}\rangle= & {\left[\int E_{-}^{b}(\boldsymbol{q}) b_{-\boldsymbol{q}} \frac{\delta}{\delta b_{-\boldsymbol{q}}} \mathrm{d} q-\int E_{+}^{b}(\boldsymbol{q}) b_{+\boldsymbol{q}} \frac{\delta}{\delta b_{+\boldsymbol{q}}} \mathrm{d} q\right]|\mathfrak{F}\rangle } \\
& +\sum_{t}\left[\int E_{t}^{\mathrm{f}}(\boldsymbol{q}) l_{t \boldsymbol{q}} \frac{\delta}{\delta l_{t \boldsymbol{q}}} \mathrm{d} q+\int \bar{E}_{t}^{\mathrm{f}}(\boldsymbol{q}) \bar{l}_{t \boldsymbol{q}} \frac{\delta}{\delta \bar{l}_{t \boldsymbol{q}}} \mathrm{d} q\right]|\mathfrak{F}\rangle \\
& +\eta^{-2} \sum_{t t^{\prime}} \int F\left(\boldsymbol{k}, \boldsymbol{q} t, \boldsymbol{q}^{\prime} t^{\prime}\right) \delta\left(\boldsymbol{k}+\boldsymbol{q}-\boldsymbol{q}^{\prime}\right)\left[\frac{\delta}{\delta b_{-\boldsymbol{k}}}+\frac{\delta}{\delta b_{+\boldsymbol{k}}}\right]\left[l_{t \boldsymbol{q}} \frac{\delta}{\delta l_{t^{\prime} \boldsymbol{q}^{\prime}}}+\bar{l}_{t \boldsymbol{q}} \frac{\delta}{\delta \bar{l}_{t^{\prime} \boldsymbol{q}^{\prime}}}\right] \mathrm{d} k \mathrm{~d} q \mathrm{~d} q^{\prime}|\mathfrak{F}\rangle \\
& +\eta^{-2} \sum_{t t^{\prime}} \int \hat{F}\left(\boldsymbol{k}, \boldsymbol{q} t, \boldsymbol{q}^{\prime} t^{\prime}\right) \delta\left(\boldsymbol{k}+\boldsymbol{q}-\boldsymbol{q}^{\prime}\right)\left[b_{-\boldsymbol{k}}-b_{+\boldsymbol{k}}\right] \frac{\delta}{\delta \bar{l}_{t \boldsymbol{q}}} \frac{\delta}{\delta l_{t^{\prime} \boldsymbol{q}^{\prime}}} \mathrm{d} k \mathrm{~d} q \mathrm{~d} q^{\prime}|\mathfrak{F}\rangle \equiv \mathscr{H}|\mathfrak{F}\rangle
\end{aligned}
$$


and by the transformation (4.36) it goes over into

$$
E|\mathfrak{5}\rangle=\exp \left(-Z_{1}\right) \mathscr{H} \exp \left(Z_{1}\right)|\mathfrak{5}\rangle .
$$

To evaluate (4.38) we have to observe that for the composite fermion and composite antifermion energies $E_{t}^{\mathrm{f}}(\boldsymbol{q})$ and $\bar{E}_{t}^{\mathrm{f}}(\boldsymbol{q})$ the relation $\bar{E}_{t}^{\mathrm{f}}(\boldsymbol{q})=-E_{t}^{\mathrm{f}}(\boldsymbol{q})$ holds as long as we have not renormalized these states by the introduction of a Dirac sea with respect to the composite particles. With this relation it can easily be shown that

$$
\left[\left(\mathscr{H}_{b b}^{0}+\mathscr{H}_{l l}^{0}+\mathscr{H}_{\bar{l}}^{0}\right), \exp Z_{1}\right]_{-}=0
$$

is satisfied. Therefore the kinetic energies of the composite particles are not touched by this transformation and only the interaction terms undergo a modification. The straightforward evaluation of (4.38) then yields

$$
\begin{aligned}
E|\mathfrak{F}\rangle= & {\left[\int E_{-}^{b}(\boldsymbol{q}) b_{-\boldsymbol{q}} \frac{\delta}{\delta b_{-\boldsymbol{q}}} \mathrm{d} q-\int E_{+}^{b}(\boldsymbol{q}) b_{+\boldsymbol{q}} \frac{\delta}{\delta b_{+\boldsymbol{q}}} \mathrm{d} q\right]|\mathfrak{5}\rangle } \\
& +\sum_{t}\left[\int E_{t}^{\mathrm{f}}(\boldsymbol{q}) l_{t \boldsymbol{q}} \frac{\delta}{\delta l_{t \boldsymbol{q}}} \mathrm{d} q+\int \bar{E}_{t}^{\mathrm{f}}(\boldsymbol{q}) \bar{l}_{t \boldsymbol{q}} \frac{\delta}{\delta \bar{l}_{t \boldsymbol{q}}} \mathrm{d} q\right]|\mathfrak{5}\rangle \\
& +\eta^{-2} \sum_{t t^{\prime}} \int F\left(\boldsymbol{k}, \boldsymbol{q} t, \boldsymbol{q}^{\prime} t^{\prime}\right) \delta\left(\boldsymbol{k}+\boldsymbol{q}-\boldsymbol{q}^{\prime}\right)\left[\frac{\delta}{\delta b_{-\boldsymbol{k}}}+\frac{\delta}{\delta b_{+\boldsymbol{k}}}\right]\left[l_{t \boldsymbol{q}} \frac{\delta}{\delta l_{t^{\prime} \boldsymbol{q}^{\prime}}}+\bar{l}_{t \boldsymbol{q}} \frac{\delta}{\delta \bar{l}_{t^{\prime} \boldsymbol{q}^{\prime}}}\right] \mathrm{d} k \mathrm{~d} q \mathrm{~d} q^{\prime}|\mathfrak{5}\rangle \\
& +\eta^{-2} \sum_{t t^{\prime}} \int \hat{F}\left(\boldsymbol{k}, \boldsymbol{q} t, \boldsymbol{q}^{\prime} t^{\prime}\right) \delta\left(\boldsymbol{k}+\boldsymbol{q}-\boldsymbol{q}^{\prime}\right)\left[b_{-\boldsymbol{k}}-b_{+\boldsymbol{k}}\right] \frac{\delta}{\delta \bar{l}_{t \boldsymbol{q}}} \frac{\delta}{\delta l_{t \boldsymbol{q}}} \mathrm{d} k \mathrm{~d} q \mathrm{~d} q^{\prime} \mid(\mathfrak{5}\rangle \\
& +\eta^{-2} \sum_{t t^{\prime}} \int F\left(\boldsymbol{k}, \boldsymbol{q} t, \boldsymbol{q}^{\prime} t^{\prime}\right) \delta\left(\boldsymbol{k}+\boldsymbol{q}-\boldsymbol{q}^{\prime}\right)\left[b_{-\boldsymbol{k}}+b_{+\boldsymbol{k}}\right] \Delta(\boldsymbol{k})\left[l_{t \boldsymbol{q}} \frac{\delta}{\delta l_{t^{\prime} \boldsymbol{q}^{\prime}}}+\bar{l}_{t \boldsymbol{q}} \frac{\delta}{\delta \bar{l}_{t^{\prime} \boldsymbol{q}^{\prime}}}\right] \mathrm{d} k \mathrm{~d} q \mathrm{~d} q^{\prime}|\mathfrak{5}\rangle \\
& +\eta^{-2} \sum_{t t^{\prime}} \int F\left(\boldsymbol{k}, \boldsymbol{q} t, \boldsymbol{q}^{\prime} t^{\prime}\right) \delta\left(\boldsymbol{k}+\boldsymbol{q}-\boldsymbol{q}^{\prime}\right)\left[\frac{\delta}{\delta b_{-\boldsymbol{k}}}+\frac{\delta}{\delta b_{+\boldsymbol{k}}}\right]\left[l_{t \boldsymbol{q}} S\left(\boldsymbol{q}^{\prime}\right) \bar{l}_{t^{\prime} \boldsymbol{q}^{\prime}}-\bar{l}_{t} S\left(\boldsymbol{q}^{\prime}\right) l_{t^{\prime} \boldsymbol{q}^{\prime}}\right] \mathrm{d} k \mathrm{~d} q \mathrm{~d} q^{\prime} \mid(\mathfrak{5}\rangle \\
& +\eta^{-2} \sum_{t t^{\prime}} \int F\left(\boldsymbol{k}, \boldsymbol{q} t, \boldsymbol{q}^{\prime} t^{\prime}\right) \delta\left(\boldsymbol{k}+\boldsymbol{q}+\boldsymbol{q}^{\prime}\right)\left[b_{-\boldsymbol{k}}+b_{+\boldsymbol{k}}\right] \Delta(\boldsymbol{k})\left[l_{t \boldsymbol{q}} S\left(\boldsymbol{q}^{\prime}\right) \bar{l}_{t^{\prime} \boldsymbol{q}^{\prime}}-\bar{l}_{t \boldsymbol{q}} S\left(\boldsymbol{q}^{\prime}\right) l_{t^{\prime} \boldsymbol{q}^{\prime}}\right] \mathrm{d} k \mathrm{~d} q \mathrm{~d} q^{\prime}|\mathfrak{5}\rangle \\
& +\eta^{-2} \sum_{t t^{\prime}} \int \hat{F}\left(\boldsymbol{k}, \boldsymbol{q} t, \boldsymbol{q}^{\prime} t^{\prime}\right) \delta\left(\boldsymbol{k}+\boldsymbol{q}-\boldsymbol{q}^{\prime}\right)\left[b_{-\boldsymbol{k}}-b_{+\boldsymbol{k}}\right]\left[l_{t \boldsymbol{q}} S(\boldsymbol{q}) \frac{\delta}{\delta l_{t^{\prime} \boldsymbol{q}^{\prime}}}+\bar{l}_{t^{\prime} \boldsymbol{q}^{\prime}} S\left(\boldsymbol{q}^{\prime}\right) \frac{\delta}{\delta \bar{l}_{t \boldsymbol{q}}}\right] \mathrm{d} k \mathrm{~d} q \mathrm{~d} q^{\prime}|\mathfrak{5}\rangle \\
& +\eta^{-2} \sum_{t t^{\prime}} \int \hat{F}\left(\boldsymbol{k}, \boldsymbol{q} t, \boldsymbol{q}^{\prime} t^{\prime}\right) \delta\left(\boldsymbol{k}+\boldsymbol{q}+\boldsymbol{q}^{\prime}\right)\left[b_{-\boldsymbol{k}}-b_{+\boldsymbol{k}}\right] l_{t \boldsymbol{q}} S(\boldsymbol{q}) S\left(\boldsymbol{q}^{\prime}\right) \bar{l}_{t^{\prime} \boldsymbol{q}^{\prime}} \mathrm{d} k \mathrm{~d} q \mathrm{~d} q^{\prime} \mid(\mathfrak{5}\rangle
\end{aligned}
$$

which is the analogon to (1.9). The Eqs. (1.9) and (4.40) become equivalent if the formfactor $F$ and $\hat{F}$ degenerate to merely algebraic quantities which do not depend on $\boldsymbol{k}, \boldsymbol{q}$ and $\boldsymbol{q}^{\prime}$. In this case we have local interactions and both theories coincide. This was shown in III to occur for sufficiently low energies. On the other hand, nontrivial formfactors appear for sufficiently high energies as can be seen from (4.20), (4.21), (4.22) and (4.29). Hence, in the high energy range the quantum theory of composite bosons and fermions deviates from a phenomenolog- ical boson-fermion coupling theory with local coupling and pointlike particles. This of course has to be expected and the only question is whether the quantum theory defined by (4.40) satisfies all essential requirements of a reasonable quantum theory or not. If it does so, then (4.40) can be used for calculations of physical processes, i. e. can be used for comparison with experiment. If not, one has to return to the original spinorfield theory in order to calculate physical processes in high energy ranges. Postponing 
the discussion of this question to the next section we finally will perform in this section a first step in the investigation of the properties of the high energy formfactors.

In order to calculate these formfactors explicitly, we consider a perturbation calculation for (4.40) and evaluate corresponding matrix elements for first order elementary scattering processes in special frames of reference. None of these first order processes can really occur since energy and momentum conservation cannot be satisfied simultaneously. Rather these processes are only admitted to occur as intermediate steps of real processes. With respect to the determination of the formfactors this restriction is, however, irrelevant.

i) The first interaction term in (4.40) describes processes where among other ones a (composite) fermion absorbs a (composite) boson. If a perturbation calculation is used to derive functional scattering states, in analogy to the perturbation theory in ordinary spaces we have first to calculate the matrix elements of the interaction terms between the free (composite) particle functional states of (4.40). In our case the initial state contains a fermion of momentum $\boldsymbol{v}$ and spin $s$ and a (positive energy) boson of momentum $\boldsymbol{p}$, while the intermediate (final) state contains only one fermion of momentum $\boldsymbol{l}^{\prime}$ and spin $s^{\prime}$ but no boson. If $|0\rangle$ denotes the functional vacuum state, then these states are given by

$$
\begin{aligned}
& \left|\mathfrak{G}_{\text {in }}\right\rangle:=|\boldsymbol{p}, \boldsymbol{v}, s\rangle \equiv b_{+\boldsymbol{p}} l_{s \boldsymbol{v}}|0\rangle ; \\
& \left\langle\mathfrak{G}_{\mathrm{f}}\right|:=\left\langle{ }^{\prime}, s^{\prime}\right| \equiv\langle 0| \frac{\delta}{\delta l_{s^{\prime} \boldsymbol{v}^{\prime}}},
\end{aligned}
$$

where the functional sources correspond to creation operators in ordinary perturbation theory, while their derivatives correspond to annihiliation operators. Then the matrix element of the first interaction term of (4.40) between these two states reads

$$
\begin{array}{r}
\left\langle\mathfrak{G}_{\mathrm{f}}\right| \eta^{-2} \sum_{t t^{\prime}} \int F\left(\boldsymbol{k}, \boldsymbol{q} t, \boldsymbol{q}^{\prime} t^{\prime}\right) \delta\left(\boldsymbol{k}+\boldsymbol{q}-\boldsymbol{q}^{\prime}\right) \frac{\delta}{\delta b_{+\boldsymbol{k}}} \\
\cdot l_{t \boldsymbol{q}} \frac{\delta}{\delta l_{t^{\prime} \boldsymbol{q}^{\prime}}} \mathrm{d} k \mathrm{~d} q \mathrm{~d} q^{\prime}\left|\mathfrak{G}_{\text {in }}\right\rangle
\end{array}
$$

where we have already omitted all those terms whose contributions vanish. A straightforward calculation yields

$$
(4.42)=\eta^{-2} \sum_{t} F(\boldsymbol{p},(\boldsymbol{v}-\boldsymbol{p}), t, \boldsymbol{v}, s) \delta_{s s^{\prime}} \delta\left(\boldsymbol{v}^{\prime}-\boldsymbol{v}+\boldsymbol{p}\right)
$$

Going into the rest frame $v \equiv 0$ of the initial fermion we obtain the formfactor $F(\boldsymbol{p},-\boldsymbol{p}, t, 0, s)$. As the spin part of this formfactor is a function bounded everywhere, the high energy behavior of $F$ is governed by the scalar part (4.22). The evaluation of this part for high energy processes, i.e. $\boldsymbol{p} \rightarrow \infty$ runs along the same lines as the calculation of the interaction terms themselves. In particular, we make use of the definition of normalized functions (2.52) for this evaluation. Then a rough estimate yields for the scalar part

$$
f(\boldsymbol{p},-\boldsymbol{p}, 0) \approx M^{3} E(\boldsymbol{p})^{-3 / 2} \text { for } \boldsymbol{p} \rightarrow \infty
$$

If this result is extrapolated to the low energy limit then we have

$$
f(\boldsymbol{p},-\boldsymbol{p}, 0) \approx M^{3 / 2} \text { for } \boldsymbol{p} \rightarrow 0 .
$$

This value coincides with the low energy value of the calculation in III for this formfactor. It thus confirms the result obtained there, since the derivation of (4.45) was done by means of more realistic wave functions.

ii) The second interaction term in (4.40) describes processes where among other ones a (composite) fermion and a (composite) antifermion annihilate and a (composite) boson is created. The initial and final states are thus given by

$$
\begin{aligned}
& \left\langle\mathfrak{W}_{\text {in }}\right\rangle:=\left|\boldsymbol{v}^{\prime}, s^{\prime}, \boldsymbol{v}, s\right\rangle \equiv l_{s^{\prime}, \boldsymbol{v}^{\prime}} \bar{l}_{s \boldsymbol{v}}|0\rangle ; \\
& \left\langle\mathfrak{G}_{\mathrm{f}}\right|:=\langle\boldsymbol{p}| \equiv\langle 0| \frac{\delta}{\delta b_{+\boldsymbol{p}}}
\end{aligned}
$$

and the corresponding matrix element reads

$$
\begin{aligned}
\left\langle\mathfrak{G}_{\mathrm{f}}\right| \eta^{-2} & \sum_{t t^{\prime}} \int \hat{F}\left(\boldsymbol{k}, \boldsymbol{q} t, \boldsymbol{q}^{\prime} t^{\prime}\right) \\
\cdot & \delta\left(\boldsymbol{k}+\boldsymbol{q}-\boldsymbol{q}^{\prime}\right) b_{+\boldsymbol{k}} \frac{\delta}{\delta \bar{l}_{t \boldsymbol{q}}} \frac{\delta}{\delta l_{t^{\prime} \boldsymbol{q}^{\prime}}} \mathrm{d} k \mathrm{~d} q \mathrm{~d} q^{\prime}\left|\mathfrak{G}_{\text {in }}\right\rangle,
\end{aligned}
$$

where we have already omitted all those terms whose contributions vanish. A straightforward calculation yields

$$
(4.47)=\eta^{-2} \sum_{s s^{\prime}} \hat{F}\left(\boldsymbol{p}, \boldsymbol{v}, s, \boldsymbol{v}^{\prime}, s^{\prime}\right) \delta\left(\boldsymbol{p}+\boldsymbol{v}+\boldsymbol{v}^{\prime}\right) .
$$

Going to the rest frame $v^{\prime} \equiv 0$ of the initial fermion we obtain the formfactor $\hat{F}\left(-\boldsymbol{p}, \boldsymbol{p}, s, 0, s^{\prime}\right)$. As the spin part of this formfactor is a function bounded everywhere the high energy behavior of $\hat{F}$ is governed by the scalar part of $\hat{F}$. In contrast to $F$ for $\hat{F}$ both these parts, however, cannot be separated. 
Using the same techniques with respect to the evaluation of $\hat{F}$ as were used for the evaluation of $F$ we obtain a rough estimate in the high energy limit by the expression

$$
\begin{gathered}
\hat{F}\left(-\boldsymbol{p}, \boldsymbol{p}, s, 0, s^{\prime}\right) \approx K^{s s^{\prime}}(\boldsymbol{p}, 0,0,-\boldsymbol{p}) M^{3} E(\boldsymbol{p})^{-3 / 2} \\
\text { for } \boldsymbol{p} \rightarrow \infty .
\end{gathered}
$$

If this result is extrapolated to the low energy limit, then we have

$$
\begin{array}{r}
\hat{F}\left(-\boldsymbol{p}, \boldsymbol{p}, s, 0, s^{\prime}\right) \approx K^{s s^{\prime}}(\boldsymbol{p}, 0,0,-\boldsymbol{p}) M^{3 / 2} \\
\text { for } \boldsymbol{p} \rightarrow 0 .
\end{array}
$$

This value coincides with the low energy value of the calculation in III for this formfactor. It thus confirms the result obtained there, since the derivation of (4.45) was done by means of more realistic wave functions.

In ordinary quantum field theory formfactors were introduced ad hoc in order to remove ultraviolet divergencies. In the unified nonlinear spinorfield model such formfactors appear as a consequence of the weak mapping with respect to composite particle states. As the ultraviolet divergencies are caused by the local interactions of point particles, one should expect that the use of composite, i.e. extended particles automatically resolves these difficulties. Although the calculations of the formfactors given here are done in special frames of reference their results indicate that this expectation will be justified in more detailed investigations.

\section{Relativistic Invariance, Causality and Unitarity}

In relativistic quantum field theory relativistic invariant representations play a preferred role. If other representations are used this has to be justified. In particular the energy representation of the unified nonlinear spinorfield model used so far needs justification.

Energy is the fourth component of the fourmomentum vector. Hence an energy representation cannot be a relativistic invariant representation. Due to the multi-time dependence of the field theoretic relativistic amplitudes this situation becomes even worse in quantum field theory: In order to evaluate the quantum field theoretic energy representation which was introduced in [6] and which is basic for all further developments of the unified nonlinear spinorfield model, the limit to equal times in the multi-time amplitudes of the generating functional has to be performed. In nonrelativistic quantum mechanics where this limit was first applied [96] this procedure is unique. In quantum field theory, however, the simultaneity concept depends on the frame of reference. Events which are located at different space points and which occur simultaneously in one frame of reference are in general not simultaneous in another frame of reference with respect to relativistic transformations between both frames. In addition, after carrying out the limit procedure the former multitime amplitudes lose their relativistic transformation properties due to the impossibility to associate an individual time to each space coordinate separately. Thus, while in classical field theory the energy component is not relativistic invariant but still can at least be properly transformed, in quantum field theory even the transformation properties are lost. Before continuing to apply the energy representation, this strange feature has to be discussed.

Without proof in I we made the following assertion: Between the multi-time amplitudes and the amplitudes of the energy representation a biunique correspondence exists.

Taking for granted this assertion, we can overcome the above mentioned difficulties: Whenever relativistic invariance and transformation properties are needed, the energy functional states can uniquely be continued into the multi-time region to give the relativistic invariant multi-time generating functionals and from the latter all relevant informations can be obtained. From this point of view the total content of the theory is already contained in the energy representation, apart from the transformation properties which must be deduced from a continuation into the multi-time region. In this way, the energy representation is the quantum version of the Hamilton formulation of a field theory which determines this field theory completely but does not show explicit relativistic invariance.

With respect to practical calculations it is, of course, a matter of convenience to avoid the continuation of the energy representation into the multi-time region. In particular it might be easier to project the abstract multi-time generating functionals onto to the various energy representations in different frames of reference. But the crucial point for all these mapping techniques is the validity of 
the above mentioned assertion. It is thus of importance to give an explicit proof of this assertion.

In I it was indicated that the functional equations should be used for such a proof. A more detailed study, however, shows that it is hard to overcome this problem directly in full generality. We thus restrict ourselves to prove this assertion for the case of two-point functions which are assumed to be solutions of the functional cluster equation (2.1). For this case the following theorem holds:

Theorem: The two-particle solutions of the energy functional equation (2.1) possess a biunique relation to the corresponding multi-time solutions of the relativistic invariant functional cluster equation, and the continuation of the one-time functional energy eigenstates into the multi-time region is identical with the last step of the complete solution procedure of the latter equation.

Proof: According to I the relativistic invariant (multi-time) cluster equation reads

$$
\begin{aligned}
& {\left[G_{a_{1} a_{2}}^{\mu} \delta_{i_{1} i_{2}} \partial_{\mu}-m_{i_{1} a_{2}} \delta_{a_{1} a_{2}} \delta_{i_{1} i_{2}}\right] \partial_{i_{2} a_{2}}(x)|\mathfrak{G}\rangle} \\
& =-x_{i_{1}} W_{a_{1} a_{2} a_{3} a_{4}} \sum_{i_{2} i_{3} i_{4}} \int F_{i_{2} a_{2} i a}\left(x-x^{\prime}\right) j_{i a}\left(x^{\prime}\right) \mathrm{d}^{4} x^{\prime} \\
& \cdot \partial_{i_{3} a_{3}}(x) \partial_{i_{4} a_{4}}(x)|\mathfrak{F}\rangle
\end{aligned}
$$

with $i \equiv$ auxiliary field index, $a \equiv$ spinor-superspinor index. The two-point amplitudes are defined by the functional states

$$
\begin{aligned}
|\mathfrak{\mho}\rangle=\frac{1}{2} \int \varphi_{h_{1} c_{1} h_{2} c_{2}}\left(y_{1}, y_{2}\right) j_{h_{1} c_{1}}\left(y_{1}\right) \\
\cdot j_{h_{2} c_{2}}\left(y_{2}\right)|0\rangle \mathrm{d}^{4} y_{1} \mathrm{~d}^{4} y_{2} .
\end{aligned}
$$

Substitution of (5.2) into (5.1) and subsequent projection of this equation into the coordinate space yield the following equations

$$
\begin{aligned}
& \left(G_{a_{1} a_{1}^{\prime}}^{\mu} \partial_{\mu x}-m_{i_{1} a_{1}} \delta_{a_{1} a_{1}^{\prime}}\right) \varphi_{i_{1} a_{1} i a}(x, y) \\
& \quad=x_{i_{1}} W_{a_{1} a_{2} a_{3} a_{4}} \sum_{i_{2} i_{3} i_{4}} F_{i_{2} a_{2} i a}(x-y) \varphi_{i_{3} a_{3} i_{4} a_{4}}(x, x) \\
& \left(G_{a a^{\prime}}^{\mu} \partial_{\mu y}-m_{i a} \delta_{a a^{\prime}}\right) \varphi_{i_{1} a_{1} i a^{\prime}}(x, y) \\
& =x_{i} W_{a a_{2} a_{3} a_{4}} \sum_{i_{2} i_{3} i_{4}} F_{i_{1} a_{1} i_{2} a_{2}}(x-y) \varphi_{i_{3} a_{3} i_{4} a_{4}}(y, y)
\end{aligned}
$$

We now introduce center of mass coordinates by the transformation

$$
\begin{array}{ll}
z=\frac{1}{2}(x+y) ; & \partial_{x}=\frac{1}{2} \partial_{z}+\partial_{u} ; \\
u=x-y ; & \partial_{y}=\frac{1}{2} \partial_{z}-\partial_{u},
\end{array}
$$

and decompose $\partial_{z}$ and $\partial_{u}$ into time and space components. Then (5.3) can be rewritten in the form

$$
\begin{aligned}
\left(\frac{1}{2} \partial_{0 z}\right. & \left.+\partial_{0 u}\right) \hat{\varphi}_{i b j c}(z, u) \\
+ & {\left[G_{b a_{1}}^{0-1} \boldsymbol{G}_{a_{1} a_{1}^{\prime}} \cdot\left(\frac{1}{2} \nabla_{z}+\nabla_{u}\right)-G_{b a_{1}}^{0-1} m_{i a_{1}} \delta_{a_{1} a_{1}^{1}}\right] \hat{\varphi}_{i a_{1}^{\prime} j c}(z, u) } \\
= & x_{i} G_{b a_{1}}^{0-1} W_{a_{1} a_{2} a_{3} a_{4}} \sum_{i_{2} i_{3} i_{4}} F_{i_{2} a_{2} j c}(u) \hat{\varphi}_{i_{3} a_{3} i_{4} a_{4}}(z, 0), \\
\left(\frac{1}{2} \partial_{0 z}\right. & \left.-\partial_{0 u}\right) \hat{\varphi}_{i b j c}(z, u) \\
& +\left[G_{c a}^{0-1} \boldsymbol{G}_{a a^{\prime}}\left(\frac{1}{2} \nabla_{z}-\nabla_{u}\right)-G_{c a}^{0-1} m_{j a} \delta_{a a^{\prime}}\right] \hat{\varphi}_{i b j a^{\prime}}(z, u) \\
= & -x_{j} G_{c a}^{0-1} W_{a a_{2} a_{3} a_{4}} \sum_{i_{2} i_{3} i_{4}} F_{i_{2} a_{2} i b}(-u) \hat{\varphi}_{i_{3} a_{3} i_{4} a_{4}}(z, 0)
\end{aligned}
$$

with $\varphi(x, y)=\hat{\varphi}(z, u)$. If we take the sum and the difference of both these equations and if without loss of generality we make the ansatz

$$
\hat{\varphi}(z, u)=e^{i p z} \chi(u \mid p)
$$

then Eqs. (5.5) go over into

$$
\begin{aligned}
& E \chi_{i b j c}(u p) \\
& +\left\{G_{b a_{1}}^{0-1}\left[\boldsymbol{G}_{a_{1} a_{1}^{\prime}}\left(\frac{1}{2} i \boldsymbol{p}+\nabla_{u}\right)-m_{i a_{1}} \delta_{a_{1} a_{1}^{\prime}}\right] \delta_{c a^{\prime}}\right. \\
& \left.+G_{c a^{\prime}}^{0-1}\left[\boldsymbol{G}_{a a^{\prime}}\left(\frac{1}{2} i \boldsymbol{p}-\nabla_{u}\right)-m_{j a} \delta_{a a^{\prime}}\right] \delta_{b a_{1}^{\prime}}\right\} \chi_{i a_{1}^{\prime} j a^{\prime}}(u p) \\
& =\sum_{i_{2} i_{3} i_{4}}\left\{x_{i} G_{b a_{1}}^{0-1} W_{a_{1} a_{2} a_{3} a_{4}} F_{i_{2} a_{2} j a^{\prime}}(u) \delta_{c a^{\prime}}\right. \\
& \left.\quad-x_{j} G_{c a^{\prime}}^{0-1} W_{a a_{2} a_{3} a_{4}} F_{i_{2} a_{2} i a^{\prime}}(-u) \delta_{b a^{\prime}}\right\} \chi_{i_{3} a_{3} i_{4} a_{4}}(0 p)
\end{aligned}
$$

and

$$
\begin{aligned}
& 2 \partial_{0 u} \chi_{i b j c}(u p) \\
& +i G_{b a_{1}}^{0-1}\left[\boldsymbol{G}_{a_{1} a_{1}^{\prime}}\left(\frac{1}{2} i \boldsymbol{p}+\nabla_{u}\right)-m_{i a_{1}} \delta_{a_{1} a_{1}^{\prime}}\right] \dot{\delta}_{c a^{\prime}} \\
& \left.\quad+G_{c a}^{0-1}\left[\boldsymbol{G}_{a a^{\prime}}\left(\frac{1}{2} i \boldsymbol{p}-\nabla_{u}\right)-m_{j a} \dot{\delta}_{a a^{\prime}}\right] \dot{\delta}_{b a_{1}^{\prime}}\right\} \chi_{i a_{1}^{\prime} j a^{\prime}}(u p) \\
& =\sum_{i_{2} i_{3} i_{4}}\left\{x_{i} G_{b a_{1}}^{0-1} W_{a_{1} a_{2} a_{3} a_{4}} F_{i_{2} a_{2} j a^{\prime}}(u) \delta_{c a^{\prime}}\right. \\
& \left.\quad+x_{j} G_{c a}^{0-1} W_{a a_{2} a_{3} a_{4}} F_{i_{2} a_{2} i a^{\prime}}(-u) \delta_{b a^{\prime}}\right\} \chi_{i_{3} a_{3} i_{4} a_{4}}(0 \mid p) .
\end{aligned}
$$

Writing for brevity $\chi(u) \equiv \chi(u \mid p)$ and separating time and space coordinates we can perform the limit $u_{0} \rightarrow 0$ in (5.7) and obtain for (5.7) and (5.8) in a formal notation

$$
\begin{aligned}
& E \chi(\boldsymbol{u}, 0)+H_{1}\left(\nabla_{u}\right) \chi(\boldsymbol{u}, 0)+H_{2}(\boldsymbol{u}, 0) \chi(0)=0, \\
& \frac{\partial}{\partial t} \chi(\boldsymbol{u}, t)+\hat{H}_{1}\left(\nabla_{u}\right) \chi(\boldsymbol{u}, t)+\hat{H}_{2}(\boldsymbol{u}, t) \chi(0)=0,
\end{aligned}
$$


where $t \equiv u_{0}$ and $H_{i}$ and $\hat{H}_{i}$ are defined by the corresponding expressions of (5.7) and (5.8).

Equation (5.9) is identical with the energy representation (2.1) for two-point clusters. We thus assume that we have solved (5.9) and that $\chi_{E} \equiv$ $\chi(\boldsymbol{u}, 0)$ together with $E$ are known quantities. By means of this assumption, (5.10) can be converted into an integral equation of the form

$\chi(\boldsymbol{u}, t)=-\int_{0}^{t} \hat{H}_{1}\left(\nabla_{u}\right) \chi(\boldsymbol{u}, \tau) \mathrm{d} \tau+R(\boldsymbol{u}, t)$

with

$R(\boldsymbol{u}, t):=\chi(\boldsymbol{u}, 0)-\int_{0}^{t} \hat{H}_{2}(\boldsymbol{u}, \tau) \chi(0) \mathrm{d} \tau$.

Equation (5.12) is an integral equation of the Volterra type which allows convergent solutions by iteration for all values of the coupling constant $\lambda$, in particular for $\lambda=-1$. For $t=0$ the solution $\chi(\boldsymbol{u}, t)$ of $(5.11)$ becomes

$$
\chi(\boldsymbol{u}, 0)=R(\boldsymbol{u}, 0) \equiv \chi(\boldsymbol{u}, 0)_{E} .
$$

Therefore the multi-time amplitude $\chi(\boldsymbol{u}, t)$ is uniquely determined by $\chi(\boldsymbol{u}, 0)_{E}$ and the continuation of $\chi(\boldsymbol{u}, 0)_{E}$ into the multi-time region is a part of the integration program of (5.1). Q.E.D.

Although the extension of this procedure to higher point functions and complete functionals is in principle straightforward, the more general cases need a thorough investigation which we will not perform here. In addition, the dependence of the Volterra kernel $\hat{H}_{1}$ on $\nabla_{u}$ needs further clarification.

Concerning the literature, single-time methods have been used to a wide extent in quantum field theory partly by working with the Hamilton formalism itself or with instantaneous interactions in BetheSalpeter equations, partly by deriving single-time equations from multi-time equations etc. It would exceed the scope of this paper to cite this extensive literature explicitly. It is, however, interesting to note that the many-time formalism was considered by only a few authors. Krolikowski and Rzewuski [100] gave an equivalence proof for single-time and multi-time integro-differential equations of twoparticle states which is completely different from the proof given here. Goto [101] introduced the transformation (5.4) and arrived at equations of the type (5.5) but did not further pursue a proof of equivalence as was given here. Cocho and Flores [102] used the group theoretical relation between relativistic and nonrelativistic oscillator functions to establish an isomorphism between corresponding relativistic and nonrelativistic few-particle equations. Faustov [103] investigated the relativistic transformation properties of single-time wave functions. Love [104] eliminated the relative time coordinate from a two-particle Bethe-Salpeter equation in such a way that he obtained an exact equivalent single-time equation. Feldman and Fulton [105] studied the relation between Bethe-Salpeter and Schrödinger wave functions of many particle systems for static potentials.

With regard to causality various investigations were made within the realm of nonlocal field theories. Apart from the papers of Efimov [65] and Alebastrov and Efimov [76] who claimed to have demonstrated strict causal behavior of their models, the general conclusion is that slight causality violations may occur but that they can be overcome by a suitable choice of the formfactors, cf. [20], [21], [35], [39], [54], [55], [57], [61], [63], [65], [74]. For a nonlocal coupling theory which results from a map of a unified nonlinear spinorfield within the high energy range, the problem of causal behavior is of minor interest: The underlying spinorfield theory is strictly microcausal. Whenever its maps show acausal behavior one has to improve the map or to go back to the original spinorfield dynamics. Some authors, Ascoli [106], Rzewuski [34], Nagy and Rzewuski [47] related field equations with higher order derivatives to nonlocal interactions. Such theories represent a very special class of nonlocal fields and exhibit strict causal behavior as long as no artificial unitarization procedure of the $S$-matrix is introduced. For the general treatment of higher order derivative equations this approach is not very suitable since for their quantization the decomposition theorem [3], [4] has to be applied which reduces the higher derivative equation to a coupled system of first order derivative equations with local interactions. This system is strictly microcausal and hence no contradiction can occur.

As far as unitary is concerned the situation for a weak mapping is more difficult than in the description of coupling theories by operator equations in the Heisenberg picture. While for the latter the full apparatus of canonical quantum theory can be used, the weak mapping yields only a functional state description without an underlying operator formalism. As was discussed in the introduction such a weak- 
ening of the map is of advantage in many respects and in addition even necessary, as strong mappings do not work correctly. However, in the case of the unitarity problem the treatment is more complicated as such investigations should primarily be performed in functional space and corresponding investigations with respect to unitarity in functional spaces are not available in the required specialization. Only general considerations were performed, cf. Rzewuski [107], which cannot be applied directly to the problem of interest. Hence, at present, the simplest way out of this difficulty consists in underlying the functional equations (4.40) an auxiliary operator theory. Such an operator theory is restricted to a certain frame of reference and necessarily does not satisfy all requirements of a local relativistic coupling theory. Nevertheless, it can be used to prove unitarity since in the special frame of reference under consideration the formfactors of the interaction terms are completely space-like. Therefore, the non-existence proof for the $S$-matrix of Marnelius does not apply and the $S$-matrix exists. As the state space of bound states is positive definite as

[1] H. Stumpf, Physica 114A, 184 (1982).

[2] D. Grosser and T. Lauxmann, J. Phys. G 8, 1505 (1982).

[3] H. Stumpf, Z. Naturforsch. 37 a, 1295 (1982).

[4] D. Grosser, Z. Naturforsch. 38a, 1293 (1983).

[5] H. Stumpf, Z. Naturforsch. 39a, 441 (1984).

[6] D. Grosser, B. Hailer, L. Hornung, T. Lauxmann, and H. Stumpf, Z. Naturforsch. 38a, 1056 (1983).

[7] H. Stumpf, Z. Naturforsch. 38a, 1064 (1983); 38a, 1184 (1983).

[8] H. Stumpf, Z. Naturforsch. 40 a, 14 (1985); 40 a, 183 (1985); 40 a, 294 (1985).

[9] G. Wataghin, Z. Physik 88, 92 (1934).

[10] F. Bopp, Z. Naturforsch. 1, 53 (1946).

[11] H. McManus, Proc. Roy. Soc. London 195, 323 (1948).

[12] R. P. Feynman, Phys. Rev. 74, 939 (1948); 74, 1430 (1948).

[13] L. de Broglie, Compt. Rend. Acad. Sci. 229, 157 (1949).

[14] H. Yukawa, Prog. Theor. Phys. 2, 209 (1947); 3, 205 (1948); 3, 452 (1948); Phys. Rev. 76, 300 (1949); 76, 1731 (1949); 77, 219 (1950); 80, 1047 (1950).

[15] J. Rayski, Phil. Mag. 42, 1289 (1951).

[16] P. Kristensen and C. Möller, K. Dansk, Vidensk. Selsk. Mat.-Fys. Medd. 27, No. 7 (1952).

[17] C. Hayashi, Prog. Theor. Phys. 10, 533 (1953); 11, 226 (1954).

[18] C. Bloch, K. Dansk, Vidensk. Selsk. Mat.-Fys. Medd. 27, No. 8 (1952).

[19] M. Chretien and R. E. Peierls, Nuovo Cim. 10, 668 (1953).

[20] M. E. Ebel, K. Dansk. Vidensk. Selsk. Mat.-Fys. Medd. 29, No. 2 (1954). long as no vector or tensor bound states are considered unitarity for the auxiliary $S$-matrix is guaranteed if the Hamiltonian is a Hermitian operator. Thus the unitarity problem is reduced to the Hermiticity problem of the interaction term as the kinetic energy terms are always Hermitian operators. For instance, Marnelius [78] has formulated such Hermiticity conditions for the formfactors. As we calculated corresponding formfactors in the preceding section only in a rough approximation in special frames of reference, at present their Hermiticity properties cannot be demonstrated and the solution of the unitarity problem has to be postponed to be treated in further investigations of this model.

\section{Acknowledgement}

I would like to thank Dr. D. Großer for a critical discussion of the manuscript and Dr. B. Hailer for making available to me the two- and three-particle equations in the spinorial representation of the wave functions before publication.

[21] M. Chretien and R. E. Peierls, Proc. Soc. London A 223, 468 (1954).

[22] C. Möller, Proc. Intern. Conf. Theor. Phys., Kyoto and Tokyo 1953, Science Council of Japan, Tokyo 1954.

[23] C. Bloch, Prog. Theor. Phys. 5, 606 (1950).

[24] M. Fierz, Helv. Phys. Acta 23, 412 (1950).

[25] D. R. Yennie, Phys. Rev. 80, 1053 (1950).

[26] J. Rayski, Acta Phys. Polon. 10, 300 (1951); 11, 109 (1951); 14, 337 (1955); 15, 89 (1956); 15, 407 (1956); Nuovo Cim. 6, 1231 (1956).

[27] R. Ascoli, Nuovo Cim. 9, 759 (1952).

[28] H. L. Jordan, Z. Naturforsch. 8a, 341 (1953).

[29] P. Gulmanelly, Nuovo Cim. 10, 1582 (1953).

[30] O. Hara and H. Shimazu, Prog. Theor. Phys. 5, 1055 (1950); 7, 255 (1952); 9, 137 (1953).

[31] Y. Katayama, Prog. Theor. Phys. 10, 31 (1953).

[32] W. Pauli, Nuovo Cim. 10, 648 (1953).

[33] Y. Ono, Prog. Theor. Phys. 10, 125 (1953).

[34] J. Rzewuski, Nuovo Cim. 10, 784 (1953); Acta Phys. Polon. 12, 100 (1953); 12, 14 (1953); 14, 121 (1954); Bull. Acad. Polon. Sci. Cl. III, 2, 429 (1954); 5, 393 (1957).

[35] G. Wataghin, Nuovo Cim. 10, 1602 (1953); 10, 500 (1953); 5, 689 (1957); 9, 519 (1958); 14, 1157 (1959); Ann. Inst. H. Poincaré 1, 47 (1964).

[36] P. Budini and C. Villi, Nuovo Cim. 10, 1172 (1953).

[37] S. Kamefuchi, Prog. Theor. Phys. 11, 273 (1954).

[38] H. Gaus, Z. Naturforsch. 9a, 81 (1954).

[39] E. C. G. Stueckelberg, and G. Wanders, Helv. Phys. Acta 27,667 (1954).

[40] T. Taniuty, Prog. Theor. Phys. 13, 505 (1955); 15, 19 (1956). 